IFUM 484/FT

July 1995

\title{
RENORMALIZATION OF MATTER FIELD THEORIES ON THE LATTICE AND THE FLOW EQUATION
}

\author{
M. PERNICI \\ INFN, Sezione di Milano, Via Celoria 16, I-20133 Milano, Italy \\ M. RACITI and F. RIVA \\ Dipartimento di Fisica dell'Universita' di Milano, I-20133 Milano, Italy \\ and INFN, Sezione di Milano, Via Celoria 16, I-20133 Milano, Italy
}

\begin{abstract}
We give a new proof of the renormalizability of a class of matter field theories on a space-time lattice; in particular we consider $\phi^{4}$ and massive Yukawa theories with Wilson fermions. We use the Polchinski approach to renormalization, which is based on the Wilson flow equation; this approach is substantially simpler than the BPHZ method, applied to the lattice by Reisz. We discuss matter theories with staggered fermions. In particular we analyse a simple kind of staggered fermions with minimal doubling, using which we prove the renormalizability of a chiral sigma model with exact chiral symmetry on the lattice.
\end{abstract}

*Work supported in part by M.U.R.S.T. and EEC, Science Project SC1*_CT92/0789. 
Perturbative quantum field theories on the lattice [1] present a few difficulties that are absent in other regularization schemes; their renormalization has been studied by Reisz [2, 3, 4] in the BPHZ approach [5, 6]. Beyond the usual technical difficulties of the BPHZ approach, requiring an analysis of the topological structure of the Feynman graphs, there are those specific to lattice theories, due to the periodicity of the Green functions in momentum space. In the first place, it is non-trivial to prove a power-counting theorem on the lattice [2], since the Feynman integrands are not rational functions of the momenta, but of trigonometric functions of the momenta. In the second place one has to show that the subtraction procedure can be implemented with counterterms which are local on the lattice.

In lattice gauge theories there is an additional difficulty, the presence of an infinite number of irrelevant interaction terms in the bare action; Reisz [四] has shown that these vertices do not modify the renormalized Green functions computed in the continuum.

In theories containing fermions there is the doubling problem for fermions on the lattice [7, 8]; introducing the Wilson term [9] in the fermionic action the doublers decouple in the continuum limit, but chiral symmetry is broken in a hard way by the Wilson term. Reisz's results apply to Wilson fermions [2, 3, 4, 10]; these results have not been extended to fermionic models with doublers. While chiral invariance is not maintained by Wilson fermions, it is possible to construct models with doublers having chiral invariance on the lattice, for instance with the Kogut-Susskind [11] staggered fermions, or with simpler versions of staggered fermions [12, 13]; it has not yet been shown that these models are renormalizable.

A simple approach to renormalization in continuum quantum field theory has been initiated by Polchinski [14], in the spirit of the Wilson renormalization group [15]; this approach has been further simplified and improved in [16, 17, 18]. One studies an effective action which, besides the ultraviolet cut-off $\Lambda_{0}$, has an 'infrared' cut-off $\Lambda$. The effective action satisfies perturbatively a linear differential equation in $\Lambda$, called the flow equation. One can impose mixed boundary conditions on the effective action, fixing the relevant terms of the effective action at a renormalization scale $\Lambda=\Lambda_{1}$, while the irrelevant terms are fixed at a scale $\Lambda=\Lambda_{0}$.

In this paper we study the renormalizability of scalar and Yukawa theories on the lattice; we adapt the Polchinski approach to the lattice, introducing an 'infrared' cut-off $\Lambda$, which restricts the propagators on a periodic band in momentum space, at a distance of the order of $\Lambda$ from the poles of the propagators.

We extend Reisz's proof of renormalizability to a class of Yukawa theories with a simple kind of staggered fermions [12, 13].

In the first section we study the massive $\phi^{4}$ field theory on a hypercubic lattice; we 
prove that it is multiplicatively renormalizable, using the flow equation for the amputated connected Green functions; we show that the addition of irrelevant terms to the action does not change the renormalized Green functions.

In the second section we prove the renormalizability of a general class of matter field theories on a lattice including scalars, fermions and auxiliary fields.

In the third section we apply these results to Wilson fermions and a simple kind of staggered fermions with minimal doubling; using the latter we prove the renormalizability of a chiral sigma model with explicit chiral invariance on a hypercubic lattice. The proof is obtained by rewriting the model on a reduced lattice, on which the results of the previous section apply.

\section{Renormalization of the massive $\phi^{4}$ model on a hy- percubic lattice.}

\subsection{Introduction.}

Consider an infinite space-time hypercubic lattice with lattice spacing $a$ and sites in $x_{\mu}=n_{\mu} a$, where $n_{\mu}$ are integers, $\mu=1, \ldots, 4$. The bare action is

$$
S=\frac{a^{2}}{2} \sum_{x, \mu} \phi_{x}^{(0)}\left(2 \phi_{x}^{(0)}-\phi_{x+\hat{\mu}}^{(0)}-\phi_{x-\hat{\mu}}^{(0)}\right)+a^{4} \sum_{x}\left(\frac{m^{(0) 2}}{2} \phi_{x}^{(0) 2}+\frac{g^{(0)}}{4 !} \phi_{x}^{(0) 4}\right)+S_{i r r}
$$

where $\phi_{x}^{(0)}$ is the bare field; $m^{(0)}$ and $g^{(0)}$ are the bare parameters. $S_{i r r}$ is an irrelevant term, which will be specified below; it will be chosen in such a way that the action preserves the hypercubic space-time symmetry and the discrete symmetry $\phi_{x}^{(0)} \rightarrow-\phi_{x}^{(0)}$.

The action reads, in momentum space,

$$
S=\frac{1}{2} \int_{k} \phi^{(0)}(-k)\left(\hat{k}^{2}+m^{(0) 2}\right) \phi^{(0)}(k)+\frac{g^{(0)}}{4 !} \int_{k_{1}, \ldots, k_{4}}(2 \pi)^{4} \delta_{P}^{4}\left(\sum_{1}^{4} k_{i}\right) \prod_{i=1}^{4} \phi^{(0)}\left(k_{i}\right)+S_{i r r}
$$

where $\hat{k}_{\mu}=\frac{2}{a} \sin \frac{k_{\mu} a}{2}, \quad \delta_{P}^{4}(k)=\frac{a^{4}}{(2 \pi)^{4}} \sum_{x} e^{-i k x}$ is the periodic delta function, $\int_{k} \equiv \int_{-\frac{\pi}{a}}^{\frac{\pi}{a}} \frac{d^{4} k}{(2 \pi)^{4}}$.

The Fourier transform of $\phi_{x}$ is $\phi(k)=\frac{a^{4}}{(2 \pi)^{4}} \sum_{x} e^{-i k x} \phi_{x}$, and $\phi_{x}=\int_{k} \phi(k) e^{i k x}$.

The Fourier transform $\phi(k)$ and the kinetic operator $\hat{k}^{2}$ are periodic under $k_{\mu} \rightarrow$ $k_{\mu}+\frac{2 \pi}{a}$. The momenta can be restricted to the Brillouin zone $\left|k_{\mu}\right| \leq \frac{\pi}{a}$, provided one requires momentum conservation at the vertices only modulo $\frac{2 \pi}{a}$. 


$$
\Lambda_{0} \equiv \frac{\pi}{a}
$$

is a kind of ultraviolet cut-off on the cartesian components of the momenta.

In terms of the renormalized field $\phi_{x}=Z^{-\frac{1}{2}} \phi_{x}^{(0)}$ the action becomes

$$
S[\phi]=\frac{1}{2} \int_{k} \phi(-k)\left(\hat{k}^{2}+m^{2}\right) \phi(k)+S_{I}[\phi]
$$

with

$$
S_{I}[\phi]=\frac{1}{2} \int_{k} \phi(-k)\left(c_{1}+c_{2} \hat{k}^{2}\right) \phi(k)+\frac{c_{3}}{4 !} \int_{k_{1}, k_{2}, k_{3}, k_{4}}(2 \pi)^{4} \delta_{P}^{4}\left(\sum_{1}^{4} k_{i}\right) \prod_{i=1}^{4} \phi\left(k_{i}\right)+S_{i r r}[\phi]
$$

where

$$
c_{1}=Z m^{(0) 2}-m^{2} ; \quad c_{2}=Z-1 ; \quad c_{3}=g^{(0)} Z^{2}
$$

In terms of the loop expansion parameter $\hbar$ the renormalization constants $c_{i}$ have the series expansion $c_{i}=g \delta_{i, 3}+\sum_{l=1}^{\infty} \hbar^{l} c_{i}^{(l)}$. The $c_{i}^{(l)}$ 's are constants depending on $\Lambda_{0}, m, g$ and on the renormalization conditions. A standard set of renormalization conditions is, in terms of the proper vertices,

$$
\left.\Gamma_{2}\right|_{p=0}=m^{2} \quad ;\left.\quad \frac{1}{8} \sum_{\mu=1}^{4} \frac{\partial^{2} \Gamma_{2}}{\partial p_{\mu} \partial p_{\mu}}\right|_{p=0}=1 \quad ;\left.\quad \Gamma_{4}\right|_{p=0}=g
$$

The parameters $m$ and $g$ are called renormalized parameters. A more general set of renormalization conditions will be specified later.

$S_{i r r}[\phi]$ contains irrelevant terms which are defined on the lattice; it will be chosen linear in a parameter $y \in[0,1], S_{i r r}[\phi]=\left.y S_{i r r}[\phi]\right|_{y=1}$. The dependence of $S_{i r r}[\phi]$ and of the Green functions on $y$ will not be explicitly indicated in the following. A typical choice (though not the most general) is $S_{i r r}[\phi]=y \sum_{x} \sum_{n=3}^{\infty} d_{2 n} a^{2 n} \phi_{x}^{2 n}$, where $d_{2 n}$, with $n \geq 3$, are independent parameters. A more general form for $S_{i r r}$ will be specified later, when we will choose the renormalization conditions. Obviously a trivial choice is $S_{i r r}=0$.

Lattice perturbation theory is done using the Feynman rules, in which the propagator is

$$
D(p)=\frac{1}{\hat{p}^{2}+m^{2}}
$$

and the vertices are contained in $S_{I}$. The dependence of $D(p)$ on the ultraviolet cut-off $\Lambda_{0}(1.3)$ is not explicitly indicated.

The integrands of the Feynman integrals are periodic functions of the momenta; it is not possible to define the degree of divergence of a graph by considering its behavior for 
very large momenta, so that the usual power-counting theorems on the continuum do not apply on the lattice. A detailed analysis of the structure of the lattice diagrams shows that it is nevertheless possible to define a lattice degree of divergence and to establish a power-counting theorem on the lattice [2].

The fact that the $\phi^{4}$ model is perturbatively renormalizable (multiplicatively) on the lattice means that, for $n \geq 2$, the $n$-point connected Green functions computed with these Feynman rules admit finite limit, order by order in $\hbar$, in the continuum limit $a \rightarrow 0$, provided the constants $c_{i}^{(l)}, i=1,2,3$ are chosen in such a way that the renormalization conditions are satisfied, and provided the irrelevant vertices contained in $S_{i r r}$ are chosen to satisfy a bound to be specified later. A proof of multiplicative renormalization of $\phi^{4}$ using BPHZ has been given by Reisz [2, 3], where it was also shown that certain irrelevant terms, like $S_{i r r}[\phi]=\sum_{x} \sum_{n=3}^{\infty} d_{2 n} a^{2 n} \phi_{x}^{2 n}$, do not change the renormalized Green functions. Reisz has also shown that there is a considerable freedom in the choice of the lattice propagator [2, 3, 4]. We will return to this point at the end of this section.

We will give another proof of these facts using an approach to renormalization started by Polchinski [14] and improved in [16].

The idea followed in [16] to study renormalization consists in introducing a class of theories, labelled by a continuous parameter $\Lambda \in\left[0, \Lambda_{0}\right]$, differing from the previous theory only for the propagator, called $D_{\Lambda}$. For $\Lambda=0$ the two propagators are equal, $D_{0}(p)=D(p)$, while for $\Lambda=\Lambda_{0}$ one has $D_{\Lambda_{0}}=0$, so that for $\Lambda=\Lambda_{0}$ perturbation theory is trivial, since the proper vertices coincide with the bare vertices. The Green functions of this class of theories satisfies a 'heat' flow equation, first considered by Wilson 15 and further studied by Polchinski [14].

Renormalization of the theory is studied investigating the 'heat' flow of the Green functions from $\Lambda=\Lambda_{0}$ to $\Lambda=0$. The theory at $\Lambda=0$ is the one in which one is really interested, that is the one with the action $(1.4-5)$.

Mixed boundary conditions are applied to determine this flow. The renormalization conditions on the relevant vertices (see e.g. the renormalization conditions (1.7)) are interpreted as boundary conditions at $\Lambda=0$; the irrelevant vertices are fixed by the boundary conditions at $\Lambda=\Lambda_{0}$.

Using the flow equations one can prove by induction inequalities on the Green functions. From these inequalities one can show that the Green functions are bounded, and finally that they converge, for $\Lambda_{0} \rightarrow \infty$.

In [16] one uses a smooth cut-off on the momenta, which vanishes below an infrared scale $\Lambda$ and above an ultraviolet scale $\Lambda_{0}$.

On the lattice the situation is somewhat different, since the Feynman integrands are 
periodic functions of the momentum, with periodicity $2 \Lambda_{0}$ (see (1.3)). Some care must be taken in defining what is a 'small momentum' or a 'large momentum'. The propagator of a field is a periodic function on the Brillouin zone; roughly speaking, a momentum is small if it is close to a maximum of the propagator; it is large if it is close to a minimum of the propagator. The maximum of the propagator (1.8) for the scalar field is at $p_{\mu}=0$ in the Brillouin zone; due to periodicity it has infinite other maxima on $\mathbf{R}^{4}$, for instance in $p_{\mu}=2 \Lambda_{0}$. On the other end the point $p_{\mu}=\Lambda_{0}$ is a minimum of the scalar propagator. Then $p_{\mu}=\Lambda_{0}$ is a 'large momentum', while $p_{\mu}=2 \Lambda_{0}$ is a 'small momentum'. The notion of region of large momenta is substituted on the lattice by the notion of periodic band at a distance of the order of $\Lambda_{0}$ from the maxima of the propagators.

In the case of the scalar propagator, such a band can be identified with a region around the boundary of the Brillouin zone. In the case of staggered fermions, which we will discuss in a later section, the propagator has a maximum in $p_{\mu}=0$ and other maxima along the border of the Brillouin zone, so the band of large momenta does not coincide with the boundary of the Brillouin zone.

We will show that there is a natural way to extend the flow equation on the lattice; the infrared cut-off $\Lambda$ regulates the distance of the periodic band mentioned above from the maxima of the propagators. For $\Lambda=0$ the band coincides with the full Brillouin zone; as $\Lambda$ increases, the band recedes from the maxima of the propagators of the fields, until for $\Lambda=\Lambda_{0}$ it becomes vanishingly small.

\subsection{The flow equation.}

Introduce an 'infrared' cut-off $\Lambda$ in the propagator, with $0 \leq \Lambda \leq \Lambda_{0}$,

$$
D_{\Lambda}(p)=\frac{K_{\Lambda}(p)}{\hat{p}^{2}+m^{2}}
$$

where, for $\Lambda>0, K_{\Lambda}(p)$ is a $C^{\infty}$ function of $\frac{\hat{p}^{2}}{\Lambda^{2}}$ which vanishes for $\frac{\hat{p}^{2}}{\Lambda^{2}} \leq \frac{16}{\pi^{2}}$, is monotonously increasing for $\frac{16}{\pi^{2}}<\frac{\hat{p}^{2}}{\Lambda^{2}}<\frac{64}{\pi^{2}}$ and is equal to 1 for $\frac{\hat{p}^{2}}{\Lambda^{2}} \geq \frac{64}{\pi^{2}}$. For $\Lambda=0$ we define $K_{0} \equiv 1$.

$K_{\Lambda}(p)$ is a periodic function on the Brillouin zone; for $\Lambda$ small, it cuts off a slightly squeezed ball around $p=0$. For $\Lambda$ close to $\Lambda_{0}$, it is non-vanishing only in a slightly squeezed ball around $p=\left(\frac{\pi}{a}, \ldots, \frac{\pi}{a}\right)$.

This propagator satisfies the following conditions

$$
D_{\Lambda_{0}} \equiv 0 \quad ; \quad D_{0}(p)=\frac{1}{\hat{p}^{2}+m^{2}} .
$$

$D_{\Lambda}$ and $K_{\Lambda}$ depend on the ultraviolet cut-off $\Lambda_{0}$; here and in the following the dependence on $\Lambda_{0}$ will not be explicitly indicated. 
Observe that $\lim _{\Lambda_{0} \rightarrow \infty} D_{\Lambda}(p)$ is rotation $(O(4))$ invariant.

The partition function is given by

$$
Z_{\Lambda}[J]=N_{\Lambda} \exp -\frac{1}{\hbar} S_{I}\left(\hbar \frac{\delta}{\delta J}\right) \exp \frac{1}{2 \hbar} \int_{p} J(-p) D_{\Lambda}(p) J(p)
$$

where the source $J(p)$ has the same support as $K_{\Lambda}(p) . N_{\Lambda}$ is the normalization constant for which $Z_{\Lambda}[0]=1$.

Actually in the case of an infinite lattice $N_{\Lambda}$ diverges; to avoid this infrared divergence we could work on a finite lattice as long as we deal with the generating functionals; soon we will work with the $n$-point connected Green functions, with $n \geq 1$, in which $N_{\Lambda}$, related to the vacuum diagrams, does not appear. At that point we could take the limit of infinite lattice. For notational semplicity we will work always with an infinite lattice.

Expanding formally $S_{I}\left(\hbar \frac{\delta}{\delta J}\right)$ in powers of $\frac{\delta}{\delta J}$, and using the identity

$$
\begin{aligned}
& e^{-\frac{1}{2 \hbar} \int_{p} J(-p) D_{\Lambda}(p) J(p)}\left[\prod_{i=1}^{n} \hbar \frac{\delta}{\delta J\left(-p_{i}\right)}\right] e^{\frac{1}{2 \hbar} \int_{p} J(-p) D_{\Lambda}(p) J(p)}= \\
& \left.\prod_{i=1}^{n}\left[\phi\left(p_{i}\right)+\hbar D_{\Lambda}\left(p_{i}\right) \frac{\delta}{\delta \phi\left(-p_{i}\right)}\right]\right|_{\phi(p)=D_{\Lambda}(p) J(p)}=\left.e^{\frac{\hbar}{2} \int_{p} \frac{\delta}{\delta \phi(-p)} D_{\Lambda}(p) \frac{\delta}{\delta \phi(p)}} \prod_{i=1}^{n} \phi\left(p_{i}\right)\right|_{\phi(p)=D_{\Lambda}(p) J(p)}
\end{aligned}
$$

holding when $p_{1}, \ldots, p_{n}$ are within the support of $K_{\Lambda}(p)$, we get

$$
Z_{\Lambda}[J]=\left.N_{\Lambda} e^{\frac{1}{2 \hbar} \int_{p} J(-p) D_{\Lambda}(p) J(p)} e^{\frac{\hbar}{2} \int_{p} \frac{\delta}{\delta \phi(-p)} D_{\Lambda}(p) \frac{\delta}{\delta \phi(p)}} e^{-\frac{1}{\hbar} S_{I}(\phi)}\right|_{\phi(p)=D_{\Lambda}(p) J(p)}
$$

One has

$$
Z_{\Lambda}[J]=e^{\frac{1}{\hbar} W_{\Lambda}[J]}
$$

where $W_{\Lambda}[J]$ is the functional generator of the connected Green functions. Substituting (1.13) in (1.12) one gets

$$
e^{\frac{1}{\hbar} W_{\Lambda}[J]-\frac{1}{2 \hbar} \int_{p} J(-p) D_{\Lambda}(p) J(p)}=\left.N_{\Lambda} e^{\frac{\hbar}{2} \int_{p} \frac{\delta}{\delta \phi(-p)} D_{\Lambda}(p) \frac{\delta}{\delta \phi(p)}} e^{-\frac{1}{\hbar} S_{I}(\phi)}\right|_{\phi(p)=D_{\Lambda}(p) J(p)}
$$

The RHS of this equation is the functional generator of the connected Green functions, apart from the free contribution $D_{\Lambda}(p)$ of the two-point function. In the following we will study the amputated connected Green functions, which are amputated of the propagators $D_{\Lambda}\left(p_{i}\right)$ attached to the external legs. The functional generator for these Green functions, apart from the 2-point tree-level contribution, will be called $V_{\Lambda}[\phi]$, and it satisfies

$$
e^{+\frac{1}{\hbar} V_{\Lambda}[\phi]}=N_{\Lambda} e^{\frac{\hbar}{2} \int_{p} \frac{\delta}{\delta \phi(-p)} D_{\Lambda}(p) \frac{\delta}{\delta \phi(p)}} e^{-\frac{1}{\hbar} S_{I}(\phi)} .
$$

$V_{\Lambda}[\phi]$ has the Volterra expansion

$$
V_{\Lambda}[\phi]=\ln N_{\Lambda}+\sum_{n=1}^{\infty} \frac{1}{n !} \int_{k_{1}, \ldots, k_{n}} \phi\left(-k_{1}\right) \ldots \phi\left(-k_{n}\right)(2 \pi)^{4} \delta_{P}^{4}\left(\sum_{1}^{n} k_{i}\right) V_{\Lambda, n}\left(k_{1}, \ldots, k_{n-1}\right)
$$


$V_{\Lambda, n}\left(k_{1}, \ldots, k_{n-1}\right)$ is symmetric under permutations of $k_{1}, \ldots, k_{n}$, where $k_{n}=-\sum_{i=1}^{n-1} k_{i}$, and it is periodic under $k_{i \mu} \rightarrow k_{i \mu}+\frac{2 \pi}{a}$ for any $i=1, \ldots, n-1$ and for any $\mu$.

As in [17, 18] we will study the flow equation perturbatively in $\hbar$. The amputated connected Green functions are defined as series in $\hbar, V_{\Lambda, n}=\sum_{l \geq 0} \hbar^{l} V_{\Lambda, n}^{(l)}$.

Each graph contributing to $V_{\Lambda, n}^{(l)}\left(k_{1}, \ldots, k_{n-1}\right)$ is an amputated connected graph with internal propagators $D_{\Lambda}$, and with the vertices contained in $S_{I}$. While in $(1.14-15)$ we restricted the support of $\phi(p)$ to be equal to the one of $K_{\Lambda}$, it is clear from the Feynman graph representation that the external momenta can be arbitrarily chosen. $V_{\Lambda, n}^{(l)}\left(k_{1}, \ldots, k_{n-1}\right)$ is a $C^{\infty}$ function on the Brillouin zone.

Differentiating equation (1.15) with respect to $\Lambda$ one gets the flux equation

$$
\frac{\partial V_{\Lambda}[\phi]}{\partial \Lambda}=\frac{1}{2} \int_{p} \frac{\partial D_{\Lambda}(p)}{\partial \Lambda}\left[\hbar \frac{\delta^{2} V_{\Lambda}}{\delta \phi(-p) \delta \phi(p)}+\frac{\delta V_{\Lambda}}{\delta \phi(-p)} \frac{\delta V_{\Lambda}}{\delta \phi(p)}\right]+\hbar \frac{\partial \ln N_{\Lambda}}{\partial \Lambda} .
$$

Differentiating (1.16) with respect to $\phi\left(k_{1}\right), \ldots, \phi\left(k_{n}\right)$ and setting $\phi$ to zero one gets the flux equations for the amputated connected Green functions, for $n \geq 2$ and $l \geq 1$,

$$
\begin{gathered}
\frac{\partial V_{\Lambda, n}^{(l)}}{\partial \Lambda}\left(k_{1}, \ldots, k_{n-1}\right)=\frac{1}{2} \int_{p} \frac{\partial D_{\Lambda}(p)}{\partial \Lambda} V_{\Lambda, n+2}^{(l-1)}\left(k_{1}, \ldots, k_{n-1}, p,-p\right) \\
+\sum_{P} \sum_{n_{1}+n_{2}=n+2} \sum_{l_{1}+l_{2}=l} \frac{\partial D_{\Lambda}}{\partial \Lambda}\left(\sum_{i=1}^{n_{1}-1} k_{P i}\right) V_{\Lambda, n_{1}}^{\left(l_{1}\right)}\left(k_{P 1}, \ldots, k_{P\left(n_{1}-1\right)}\right) V_{\Lambda, n_{2}}^{\left(l_{2}\right)}\left(k_{P n_{1}}, \ldots, k_{P n}\right)
\end{gathered}
$$

where $\sum_{P}$ is the sum over distinct permutations of $k_{1}, \ldots, k_{n}$, with $k_{n}=-\sum_{1}^{n-1} k_{j}$.

In the case $l=0$ the first term in the RHS is absent, and for $l=0, n=2$ the zero solution must be chosen.

Since the sums in the RHS of (1.17) satisfy $l_{i} \geq 0, n_{i} \geq 2$, for given $l$ and $n$ they contain a finite number of terms and can be solved iteratively. Indeed in the RHS of (1.17) one has $V_{\Lambda, E}^{(s)}$, with $s \leq l-1$, or with $s=l$ and $E \leq n-2$. Ordering the vertices in such a way that $V_{\Lambda, n_{1}}^{\left(l_{1}\right)}$ preceeds $V_{\Lambda, n_{2}}^{\left(l_{2}\right)}$ if $l_{1}<l_{2}$ or if $l_{1}=l_{2}$ and $n_{1}<n_{2}$, it follows that in the RHS of (1.17) there appear only vertices preceeding $V_{\Lambda, n}^{(l)}$. Therefore (1.17) is a linear differential equation in $V_{\Lambda, n}^{(l)}$.

$V_{\Lambda, n}^{(l)}$ can be determined assigning its value at a particular value of $\Lambda$. At $\Lambda=\Lambda_{0}$ equation (1.15) reduces to

$$
V_{\Lambda_{0}}[\phi]=-S_{I}[\phi]+\hbar \ln N_{\Lambda_{0}}
$$

which provides a set of boundary conditions on $V_{\Lambda, n}^{(l)}$ at $\Lambda=\Lambda_{0}$. It follows that at $\Lambda=0$ one has

$$
\begin{gathered}
V_{0,2}^{(l)}(0)=\alpha^{(l)} ; \frac{1}{8} \sum_{\mu=1}^{4} \frac{\partial^{2} V_{0,2}^{(l)}}{\partial p_{\mu} \partial p_{\mu}}(0)=\beta^{(l)} ; V_{0,4}^{(l)}(0,0,0)=\gamma^{(l)} \\
\alpha^{(0)}=0 \quad, \quad \beta^{(0)}=0 \quad, \quad \gamma^{(0)}=-g .
\end{gathered}
$$


The flux equations for $n=2$ and $n=4$ determine the three vectors $\left(\alpha^{(l)}\right),\left(\beta^{(l)}\right)$ and $\left(\gamma^{(l)}\right)$ in terms of the three vectors $\left(c_{i}^{(l)}\right), i=1,2$ and 3 given in (1.5); in perturbation theory the viceversa is also true, since in this case (1.17) is a first-order linear differential equation.

Instead of fixing the boundary conditions for the flux equation (1.17) on all the vertices at $\Lambda=\Lambda_{0}$, one can equivalently impose the renormalization conditions (1.19) on the relevant terms, while keeping fixed at $\Lambda=\Lambda_{0}$ the boundary conditions on the irrelevant terms.

The renormalization conditions (1.19) can be expressed in terms of the renormalization conditions on the proper vertices $\Gamma_{n}$, using the relations among the proper vertices and the connected Green functions. A particular case is $\alpha^{(l)}=\beta^{(l)}=\gamma^{(l)}=0$ for $l \geq 1$; in this case the renormalization conditions (1.19) are equivalent to the renormalization conditions (1.7).

From a practical point of view it is simpler to make perturbative computations with proper vertices. However the flux equations are simpler for the amputated connected Green functions than for the proper vertices, so we have chosen to prove renormalizability using the former. A proof of the renormalizability of $\phi^{4}$ using the flux equations for the proper vertices has been given in 18 .

The Green functions $\lim _{\Lambda_{0} \rightarrow \infty} V_{0, n}^{(l)}$ can be obtained from $\lim _{\Lambda_{0} \rightarrow \infty} V_{\Lambda, n}^{(l)}$, using (1.15) and the semi-group property of the heat kernel,

$$
e^{+\frac{1}{\hbar} V_{0}[\phi]}=\frac{N_{0}}{N_{\Lambda}} e^{\frac{\hbar}{2} \int_{p} \frac{\delta}{\delta \phi(-p)}\left[D_{0}-D_{\Lambda}\right](p) \frac{\delta}{\delta \phi(p)}} e^{+\frac{1}{\hbar} V_{\Lambda}[\phi]}
$$

Following the same logic as in $(1.11-15)$, we can compute $\lim _{\Lambda_{0} \rightarrow \infty} V_{0, n}^{(l)}$ in terms of Feynman graphs, in which the propagators are given by $\lim _{\Lambda_{0} \rightarrow \infty}\left[D_{0}-D_{\Lambda}\right](p)$, and the vertices are given by $\lim _{\Lambda_{0} \rightarrow \infty} V_{\Lambda, n}^{(l)}$.

Since $m \neq 0$, the support of $\left[D_{0}-D_{\Lambda}\right](p)$ is bounded; from this fact and from a bound on $V_{\Lambda, n}^{(l)}$ which we will obtain later one could easily show that, if $\lim _{\Lambda_{0} \rightarrow \infty} V_{\Lambda, n}^{(l)}$ exists, then $\lim _{\Lambda_{0} \rightarrow \infty} V_{0, n}^{(l)}$ exists. Therefore we can fix the renormalization conditions at $\Lambda=\Lambda_{1}$ instead of the conditions (1.19):

$$
V_{\Lambda_{1}, 2}^{(l)}(0)=\alpha_{1}^{(l)} ; \frac{1}{8} \sum_{\mu=1}^{4} \frac{\partial^{2} V_{\Lambda_{1}, 2}^{(l)}}{\partial p_{\mu} \partial p_{\mu}}(0)=\beta_{1}^{(l)} \quad ; \quad V_{\Lambda_{1}, 4}^{(l)}(0,0,0)=\gamma_{1}^{(l)}
$$

with $\alpha_{1}^{(0)}=\beta_{1}^{(0)}=0$ and $\gamma_{1}^{(0)}=-g$. The vectors $\alpha^{(l)}, \beta^{(l)}$ and $\gamma^{(l)}$ can be computed in function of the vectors $\alpha_{1}^{(l)}, \beta_{1}^{(l)}$ and $\gamma_{1}^{(l)}$; order by order in perturbation theory, the viceversa is also true. Therefore for $m \neq 0$ the renormalization conditions (1.21) are equivalent to the renormalization conditions (1.19). For technical reasons we will work with the former. For $m=0$ the renormalization conditions (1.21) can be imposed, but 
it is not trivial to prove the existence of the functional $V_{\Lambda}$ for $\Lambda=0$, due to the presence of infrared divergences. This issue is studied in [18, 24] using the flow equation.

\subsection{Proof of renormalizability.}

In the following we will use the symbol $P\left(x_{1}, \ldots, x_{n}\right)$ to indicate each time a different polynomial in $x_{1}, \ldots, x_{n}$, with non-negative coefficients independent of $\Lambda_{0}$.

Indicate formally by $\partial_{k}^{z} V_{\Lambda, n}^{(l)}\left(k_{1}, \ldots, k_{n-1}\right)$ the differentiation of $V_{\Lambda, n}^{(l)}\left(k_{1}, \ldots, k_{n-1}\right) z$ times with respect to $k_{i_{1} \mu_{1}}, \ldots, k_{i_{n-1} \mu_{n-1}}$, where $k_{i_{j} \mu_{j}}$ are external momenta.

Let $V\left(k_{1}, \ldots, k_{n-1}\right)$ be a differentiable function of the momenta $k_{1}, \ldots, k_{n-1}$. In the following we will use the Taylor formula

$$
V\left(k_{1}, \ldots, k_{n-1}\right)=V(0, \ldots, 0)+\left.\sum_{\mu} \sum_{i=1}^{n-1} \int_{0}^{1} d t k_{i \mu} \frac{\partial}{\partial q_{i \mu}} V\left(q_{1}, \ldots, q_{n-1}\right)\right|_{q_{i \mu}=t k_{i \mu}}
$$

Consider a function $f_{a}\left(p_{1}, \ldots, p_{n-1}\right)$ which is symmetric under permutations of $p_{1}, \ldots, p_{n}$, where $p_{n}=-\sum_{i=1}^{n-1} p_{i}$; the subscript $a$ indicates some collection of indices. Introduce the norm

$$
\|f\|_{\Lambda}=\operatorname{Max}_{\{a\}} \operatorname{Sup}_{M_{\Lambda}}\left|f_{a}\left(p_{1}, \ldots, p_{n-1}\right)\right|
$$

where $M_{\Lambda}$ is the domain satisfying the conditions $\hat{p}_{i}^{2} \leq \frac{64}{\pi^{2}} \operatorname{Max}\left(\Lambda^{2}, \eta^{2}\right)$, for any $i=1, \ldots, n$, $p_{n}=-\sum_{1}^{n-1} p_{i} ; \eta$ is a fixed quantity, whose role will become clear later. Since $\left|\hat{p}_{\mu}\right| \geq \frac{2}{\pi}\left|p_{\mu}\right|$ for $p_{\mu}$ belonging to the Brillouin zone, it follows that a bound on $\|f\|_{\Lambda}$ implies a bound on the norm with the form (1.23), with the domain $p_{i}^{2} \leq 16 \operatorname{Max}\left(\Lambda^{2}, \eta^{2}\right)$. While the norm (1.23) depends on $\Lambda_{0}$, the latter norm does not.

An useful property of the norm (1.23) is $\|f\|_{\Lambda^{\prime}} \leq\|f\|_{\Lambda^{\prime \prime}}$ for $\Lambda^{\prime} \leq \Lambda^{\prime \prime} \leq \Lambda_{0}$.

Let us now discuss more precisely the conditions to be imposed on the term $S_{\text {irr }}$ in the action (1.5). We will assume that the coefficients $S_{\Lambda_{0}, n}^{(l)}\left(k_{1}, \ldots, k_{n-1}\right)$ of the perturbative Volterra expansion of $S_{i r r}$ be $C^{\infty}$ functions on the Brillouin zone, satisfying the bounds

$$
\begin{gathered}
\left\|\partial_{k}^{z} S_{\Lambda_{0}, n}^{(0)}\right\|_{\Lambda} \leq \frac{\Lambda^{5-n-z}}{\Lambda_{0}} P\left(\ln \frac{\Lambda_{0}}{\Lambda}\right), l=0 \\
\left\|\partial_{k}^{z} S_{\Lambda_{0}, n}^{(l)}\right\|_{\Lambda} \leq \frac{\Lambda^{5-n-z}}{\Lambda_{0}} P\left(\ln \frac{\Lambda_{0}}{\Lambda_{1}}\right), l>0
\end{gathered}
$$

for any fixed $\eta$ and for $\Lambda_{1} \leq \Lambda \leq \Lambda_{0}$. These conditions, together with the vanishing of $V_{\Lambda_{0}, 2}^{(0)}=S_{\Lambda_{0}, 2}^{(0)}$, give the previously mentioned conditions on $S_{i r r}$.

Renormalization consists in showing that $\partial_{k}^{z} V_{\Lambda, n}^{(l)}$ converges and is rotation-covariant in the limit $\Lambda_{0} \rightarrow \infty$. In order to prove renormalizability we will first prove the following proposition. 
For $\Lambda_{1} \leq \Lambda \leq \Lambda_{0}$ one has

$$
\left\|\partial_{\Lambda}^{w} \partial_{y}^{v} \partial_{k}^{z} V_{\Lambda, n}^{(s)}\right\|_{\Lambda} \leq \Lambda_{0}^{-v} \Lambda^{4-n-z-w+v} P\left(\ln \frac{\Lambda}{\Lambda_{1}},\left(\ln \frac{\Lambda_{0}}{\Lambda_{1}}\right)^{v}\right)
$$

for any $s$, for $v, w=0,1, z \geq 0$ and $n \geq 2$. To prove this we will proceed by induction in the loop index.

Equation (1.25) holds for $s=0$, as can be seen by the following power counting.

Consider a tree-level amputated connected graph with $n$ external legs, $I$ internal propagators and $V_{\bar{n}}$ bare vertices with $\bar{n}$ legs; at tree level one has $\bar{n} \geq 4$.

The internal propagators satisfy the bound

$$
\left\|\partial_{\Lambda}^{w} \partial_{k}^{z} D_{\Lambda}(k)\right\| \leq \alpha \Lambda^{-2-w-z}
$$

where $\alpha$ is a positive constant.

This graph is bounded by const. $\times \Lambda^{b}$, with

$$
b=\sum_{\bar{n} \geq 4}(4-\bar{n}) V_{\bar{n}}-2 I
$$

where we used the bounds $(1.24),(1.26)$ and the fact that const. $\times \Lambda^{4-n}>\frac{\Lambda^{5-n}}{\Lambda_{0}} P\left(\ln \frac{\Lambda_{0}}{\Lambda}\right)$. $V=\sum_{\bar{n}} V_{\bar{n}}$ is the total number of vertices in the tree graph. Using the identities

$$
n+2 I=\sum_{\bar{n} \geq 4} \bar{n} V_{\bar{n}} \quad ; \quad V-I=1
$$

it follows that $b=4-n$. Differentiating $V_{\Lambda, n}^{(0)}$ with respect to $y$, the only non-vanishing contributions are those in which there is at least one irrelevant vertex $V_{\bar{n}}$, with $\bar{n} \geq 4$; using (1.24), it follows that $\left\|\partial_{y} V_{\Lambda, n}^{(0)}\right\|_{\Lambda} \leq \frac{\Lambda^{5-n}}{\Lambda_{0}} P\left(\ln \frac{\Lambda_{0}}{\Lambda}\right)$.

Using the fact that $K_{\Lambda}$ is a $C^{\infty}$ function on the Brillouin zone, it is easy to see that differentiating the tree-level Green functions with respect to an external momentum, or with respect to $\Lambda$, lowers $b$ by one. Therefore the induction hypothesis is true for $s=0$.

Convergence and rotation-covariance of $\partial_{k}^{z} V_{\Lambda, n}^{(0)}$ for $\Lambda_{0} \rightarrow \infty$ follow trivially.

Differentiating (1.17) with respect to $y$ and $k$ we get the following inequalities, for $\Lambda_{1} \leq \Lambda \leq \Lambda_{0}$

$$
\begin{aligned}
& \left\|\partial_{\Lambda} \partial_{y}^{v} \partial_{k}^{z} V_{\Lambda, n}^{(l)}\right\|_{\Lambda} \leq d_{1} \Lambda\left\|\partial_{y}^{v} \partial_{k}^{z} V_{\Lambda, n+2}^{(l-1)}\right\|_{\Lambda}+ \\
& \quad \sum_{n_{1}+n_{2}=n+2} \sum_{l_{1}+l_{2}=l} \sum_{v_{1}+v_{2}=v} \sum_{z_{1}+z_{2}+z_{3}=z} d_{z_{1}, z_{2}, z_{3}} \Lambda^{-3-z_{3}}\left\|\partial_{y}^{v_{1}} \partial_{k}^{z_{1}} V_{\Lambda, n_{1}}^{\left(l_{1}\right)}\right\|_{\Lambda}\left\|\partial_{y}^{v_{2}} \partial_{k}^{z_{2}} V_{\Lambda, n_{2}}^{\left(l_{2}\right)}\right\|_{\Lambda}
\end{aligned}
$$

where $d_{1}$ and $d_{z_{1}, z_{2}, z_{3}}$ are constants, independent of $\Lambda$ and $\Lambda_{0}$.

Assume by induction hypothesis that (1.25) holds for $s \leq l-1$; then we want to show that (1.25) holds for $s=l$. 
We will proceed in steps; in the first three steps we make a few remarks which are used in the following steps. Then we prove (1.25) in a sequence of points, each point being established by using the previous ones. This sequence consists in increasing the number of legs and going, for fixed number of legs, from irrelevant to relevant. The first point consists in proving (1.25) for $s=l, n=2, z \geq 3$, that is for the irrelevant part of the two-point function.

i) For $n+z \geq 5$ and $n>2$ one has

$$
\left\|\partial_{y}^{v} \partial_{k}^{z} V_{\Lambda, n}^{(s)}\right\|_{\Lambda} \leq \int_{\Lambda}^{\Lambda_{0}} d \Lambda^{\prime}\left\|\partial_{\Lambda^{\prime}} \partial_{y}^{v} \partial_{k}^{z} V_{\Lambda^{\prime}, n}^{(s)}\right\|_{\Lambda^{\prime}}+\frac{\Lambda^{5-n-z}}{\Lambda_{0}} P\left(\ln \frac{\Lambda_{0}}{\Lambda_{1}}\right)
$$

where we used the boundary conditions (1.24) on the irrelevant operators. Therefore if (1.25) holds for $s=l, w=1$ and $n+z \geq 5$, with $n>2$, then (1.25) holds for $s=l$, $w=0$ and $n+z \geq 5, n>2$.

ii) In the inequality (1.29), the only terms in the RHS in which there appear vertices of order $l$ are those with $n_{1} \leq n-2, n_{2} \geq 4$ or viceversa, since there are no tree-level two-point vertices. If (1.25) holds for $s=l$ up to $n-2$ external legs, then by the induction hypothesis it holds for $s=l$, with $n$ external legs and $w=1$.

iii) We will use the following inequality, holding for $\Lambda_{1} \leq \Lambda$,

$$
\left|\partial_{y}^{v} \partial_{k}^{z} V_{\Lambda, n}^{(l)}(0, \ldots, 0)\right| \leq\left|\partial_{y}^{v} \partial_{k}^{z} V_{\Lambda_{1}, n}^{(l)}(0, \ldots, 0)\right|+\int_{\Lambda_{1}}^{\Lambda} d \Lambda^{\prime}\left\|\partial_{\Lambda^{\prime}} \partial_{y}^{v} \partial_{k}^{z} V_{\Lambda^{\prime}, n}^{(l)}\right\|_{\Lambda^{\prime}}
$$

iv) The case of the two-point function can be treated in the following steps:

a) using (ii) for $n=2$, and observing that there is no term $V_{\Lambda, 2}^{(l)}$ in the RHS of (1.29), it follows that (1.25) holds for $s=l, n=2$ and $w=1$;

b) from (iii) for $n=z=2, v=0$ and $\Lambda=\Lambda_{0}$, and using the induction hypothesis, it follows that the renormalization counterterms satisfy the bound $\left|c_{2}^{(l)}\right| \leq P\left(\ln \frac{\Lambda_{0}}{\Lambda_{1}}\right)$. Using this fact it follows that (1.30) holds also for $s=l$ and $n=2, z \geq 3$. From (a) one gets (1.25) for $s=l, n=2, z \geq 3$ and $w=0$;

c) making the Taylor expansion (1.22) for $\partial_{y}^{v} \partial_{k}^{2} V_{\Lambda, 2}^{(l)}(k)$ and using (iii) it follows that, using the renormalization conditions (1.21), (a),(b), and the fact that in the Brillouin zone one has $k_{\mu} \leq \frac{\pi}{2}\left|\hat{k}_{\mu}\right|$, we get (1.25) for $s=l, n=z=2$ and $w=0$.

d) making the Taylor expansion (1.22) for $\partial_{y}^{v} \partial_{k} V_{\Lambda, 2}^{(l)}(k)$, using the hypercubic symmetry and (c) it follows that (1.25) is true for $s=l, n=2, z=1, w=0$.

e) making the Taylor expansion (1.22) for $\partial_{y}^{v} V_{\Lambda, 2}^{(l)}(k)$, using the renormalization conditions (1.21), (a,d) and (iii), it follows that (1.25) is true for $s=l, n=2, z=0, w=0$.

Therefore we have shown that the induction hypothesis is true for $s=l, n=2$.

v) Use (i) for $n=4, z \geq 1$ to get (1.25) for $s=l, n=4, z \geq 1, w=0$. Using (iii) for $n=4, z \geq 0$ in the Taylor expansion of $\partial_{y}^{v} V_{\Lambda, 4}^{(l)}\left(k_{1}, k_{2}, k_{3}\right)$ we get, using the boundary 
conditions (1.21) and the first part of (v), the fact that (1.25) holds for $s=l, n=4, z=$ $0, w=0$. Therefore the induction hypothesis holds for $s=l, n=4$.

vi) Assume that (1.25) is true for $s=l, 4 \leq n^{\prime} \leq n-2$; then using (i) and (ii) it is easy to prove (1.25) for $n$ external legs. Since (1.25) holds for $s=l, n=4$, then by induction it holds for $s=l, n>4$.

Therefore (1.25) is true for $s=l$. By induction, it is true for any $s$.

From (1.25) it follows that $V_{\Lambda, n}^{(l)}$ is bounded for $\Lambda_{0} \rightarrow \infty$; we have now to prove that $V_{\Lambda, n}^{(l)}$ converges in this limit.

Define $\tilde{D}_{z, \Lambda}(k) \equiv \lim _{\Lambda_{0} \rightarrow \infty} \partial_{k}^{z} D_{\Lambda}(k)$ and $\tilde{V}_{z, \Lambda, n}^{(l)}\left(k_{1}, \ldots, k_{n-1}\right) \equiv \lim _{\Lambda_{0} \rightarrow \infty} \partial_{k}^{z} V_{\Lambda, n}^{(l)}\left(k_{1}, \ldots, k_{n-1}\right)$.

We will now prove the existence of $\tilde{V}_{z, \Lambda, n}^{(l)}\left(k_{1}, \ldots, k_{n-1}\right)$; moreover we will show that it is a continuous function of the momenta and satisfies

$$
\begin{gathered}
\tilde{V}_{z, \Lambda, n}^{(l)}\left(k_{1}, \ldots, k_{n-1}\right)=\tilde{V}_{z, \Lambda_{1}, n}^{(l)}\left(k_{1}, \ldots, k_{n-1}\right) \\
+\frac{1}{2} \int_{\Lambda_{1}}^{\Lambda} d \Lambda^{\prime}\left\{\int \frac{d^{4} p}{(2 \pi)^{4}} \partial_{\Lambda^{\prime}} \tilde{D}_{\Lambda^{\prime}}(p) \tilde{V}_{z, \Lambda^{\prime}, n+2}^{(l-1)}\left(k_{1}, \ldots, k_{n-1}, p,-p\right)\right. \\
\left.+\hat{\sum} \partial_{\Lambda^{\prime}} \tilde{D}_{z_{3}, \Lambda^{\prime}}\left(\sum_{i=1}^{n_{1}-1} k_{P i}\right) \tilde{V}_{z_{1}, \Lambda^{\prime}, n_{1}}^{\left(l_{1}\right)}\left(k_{P 1}, \ldots, k_{P\left(n_{1}-1\right)}\right) \tilde{V}_{z_{2}, \Lambda^{\prime}, n_{2}}^{\left(l_{2}\right)}\left(k_{P n_{1}}, \ldots, k_{P n}\right)\right\}
\end{gathered}
$$

for $n+z \leq 4$ and

$$
\begin{aligned}
& \tilde{V}_{z, \Lambda, n}^{(l)}\left(k_{1}, \ldots, k_{n-1}\right)=\frac{1}{2} \int_{\Lambda}^{\infty} d \Lambda^{\prime}\left\{\int \frac{d^{4} p}{(2 \pi)^{4}} \partial_{\Lambda^{\prime}} \tilde{D}_{\Lambda^{\prime}}(p) \tilde{V}_{z, \Lambda^{\prime}, n+2}^{(l-1)}\left(k_{1}, \ldots, k_{n-1}, p,-p\right)\right. \\
& \left.+\hat{\sum} \partial_{\Lambda^{\prime}} \tilde{D}_{z_{3}, \Lambda^{\prime}}\left(\sum_{i=1}^{n_{1}-1} k_{P i}\right) \tilde{V}_{z_{1} \Lambda^{\prime}, n_{1}}^{\left(l_{1}\right)}\left(k_{P 1}, \ldots, k_{P\left(n_{1}-1\right)}\right) \tilde{V}_{z_{2}, \Lambda^{\prime}, n_{2}}^{\left(l_{2}\right)}\left(k_{P n_{1}}, \ldots, k_{P n}\right)\right\}
\end{aligned}
$$

for $n+z \geq 5$, Here and in the following of this section we will use the symbol $\hat{\sum}=$ $\sum_{P} \sum_{n_{1}+n_{2}=n+2} \sum_{l_{1}+l_{2}=l} \sum_{z_{1}+z_{2}+z_{3}=z}$, where $\sum_{P}$ is the sum over distinct permutations of $k_{1}, \ldots, k_{n}$, with $k_{n}=-\sum_{1}^{n-1} k_{j}$.

The proof of convergence is made by induction in the loop order $l$, so that we start by considering the case $l=0$; in the limit $\Lambda_{0} \rightarrow \infty$ the only bare vertex corresponds to the tree-level term $g \phi^{4}$; the propagator converges to $\tilde{D}_{\Lambda}$; a tree-level graph on the lattice converges to the corresponding tree-level graph of the continuum $g \phi^{4}$ theory and it is easy to check that $(1.31-32)$ hold.

Assume that the induction hypothesis $(1.31-32)$ is true for $s<l$. Then we want to show that it is true for $s=l$. The integrated flux equations are

$$
\begin{gathered}
\partial_{k}^{z} V_{\Lambda, n}^{(l)}\left(k_{1}, \ldots, k_{n-1}\right)=\partial_{k}^{z} V_{\Lambda_{1}, n}^{(l)}\left(k_{1}, \ldots, k_{n-1}\right)+ \\
+\frac{1}{2} \int_{\Lambda_{1}}^{\Lambda} d \Lambda^{\prime}\left\{\int_{p} \partial_{\Lambda^{\prime}} D_{\Lambda^{\prime}}(p) \partial_{k}^{z} V_{\Lambda^{\prime}, n+2}^{(l-1)}\left(k_{1}, \ldots, k_{n-1}, p,-p\right)\right. \\
\left.+\hat{\sum} \partial_{k}^{z_{3}} \partial_{\Lambda^{\prime}} D_{\Lambda^{\prime}}\left(\sum_{i=1}^{n_{1}-1} k_{P i}\right) \partial_{k}^{z_{1}} V_{\Lambda^{\prime}, n_{1}}^{\left(l_{1}\right)}\left(k_{P 1}, \ldots, k_{P\left(n_{1}-1\right)}\right) \partial_{k}^{z_{2}} V_{\Lambda^{\prime}, n_{2}}^{\left(l_{2}\right)}\left(k_{P n_{1}}, \ldots, k_{P n}\right)\right\}
\end{gathered}
$$


for $n+z \leq 4$ and

$$
\begin{aligned}
& \partial_{k}^{z} V_{\Lambda, n}^{(l)}\left(k_{1}, \ldots, k_{n-1}\right)=\partial_{k}^{z} V_{\Lambda_{0}, n}^{(l)}\left(k_{1}, \ldots, k_{n-1}\right) \\
& +\frac{1}{2} \int_{\Lambda}^{\Lambda_{0}} d \Lambda^{\prime}\left\{\int_{p} \partial_{\Lambda^{\prime}} D_{\Lambda^{\prime}}(p) \partial_{k}^{z} V_{\Lambda^{\prime}, n+2}^{(l-1)}\left(k_{1}, \ldots, k_{n-1}, p,-p\right)\right. \\
& \left.+\hat{\sum} \partial_{k}^{z_{3}} \partial_{\Lambda^{\prime}} D_{\Lambda^{\prime}}\left(\sum_{i=1}^{n_{1}-1} k_{P i}\right) \partial_{k}^{z_{1}} V_{\Lambda^{\prime}, n_{1}}^{\left(l_{1}\right)}\left(k_{P 1}, \ldots, k_{P\left(n_{1}-1\right)}\right) \partial_{k}^{z_{2}} V_{\Lambda^{\prime}, n_{2}}^{\left(l_{2}\right)}\left(k_{P n_{1}}, \ldots, k_{P n}\right)\right\}
\end{aligned}
$$

for $n+z \geq 5$.

We start by proving convergence for $n=2, z \geq 3$. The RHS of (1.34) depends only on lower perturbative order in the loop expansion, so that by the induction hypothesis its vertices converge, and the limit of the integrand exists. Furthermore, the first term in the RHS of (1.34) goes to zero in the limit. From (1.25) it follows that, for $\eta \geq \operatorname{Max}\left|k_{i}\right|$, the integrand appearing in the RHS of (1.34) is bounded by a $\Lambda_{0}$-independent integrable function of the integration variables; by the dominated convergence theorem of Lebesgue, it follows that (1.32) holds in this case.

For $n=2, z=2$ analogous considerations can be applied to the integral of the RHS of (1.33); the first term in the RHS of (1.33) is Taylor-expanded using (1.22), using the renormalization conditions (1.21) and the convergence proof for $n=2, z \geq 3$; it follows that $\partial_{k}^{2} V_{\Lambda, 2}^{(l)}$ converges. The proof is repeated for $z=1$ and $z=0$ in a similar way. For $n=4$ one realizes that the RHS of (1.34) contains $V_{\Lambda, n^{\prime}}^{\left(l^{\prime}\right)}$ with $l^{\prime}=l$ but only with $n^{\prime}=2$ and so using the previous result the proof holds again.

For $n>4$, as in the proof of (1.25), a systematic induction in $n$ starts working with (1.34).

Having proven that the vertices converge, let us now show that they are continuous in the momenta. In fact, the integrands in the RHS of (1.31) and (1.32) are continuous functions of the momenta by induction hypothesis; using (1.25) and (1.26) for suitable $\eta$ we get a momentum-independent integrable bound which is a function of the integration variables, so that a standard theorem leads to the conclusion that the RHS of equations (1.31) and (1.32) are continuous functions of the momenta.

It is now an easy task to show that the limit for $\Lambda_{0} \rightarrow \infty$ and the limits involved in the derivatives commute, that is

$$
\tilde{V}_{z, \Lambda, n}^{(l)}\left(k_{1}, \ldots, k_{n-1}\right)=\partial_{k}^{z} \lim _{\Lambda_{0} \rightarrow \infty} V_{\Lambda, n}^{(l)}\left(k_{1}, \ldots, k_{n-1}\right)
$$

We give the proof for $z=1$. From

$$
V_{\Lambda, n}^{(l)}\left(k_{1}+\Delta k, \ldots, k_{n-1}\right)-V_{\Lambda, n}^{(l)}\left(k_{1}, \ldots, k_{n-1}\right)=\int_{k_{1}}^{k_{1}+\Delta k} d p \partial_{p} V_{\Lambda, n}^{(l)}\left(p, k_{2}, \ldots, k_{n-1}\right)
$$

and by another application of the Lebesgue theorem we have

$$
\lim _{\Lambda_{0} \rightarrow \infty} \frac{V_{\Lambda, n}^{(l)}\left(k_{1}+\Delta k, \ldots, k_{n-1}\right)-V_{\Lambda, n}^{(l)}\left(k_{1}, \ldots, k_{n-1}\right)}{\Delta k}
$$




$$
=\frac{1}{\Delta k} \int_{k_{1}}^{k_{1}+\Delta k} d p \lim _{\Lambda_{0} \rightarrow \infty} \partial_{p} V_{\Lambda, n}^{(l)}\left(p, \ldots, k_{n-1}\right)
$$

where the integrand in the RHS is a continuous function, by the previous results; taking the limit $\Delta k \rightarrow 0$ one gets immediately the assertion.

From (1.25) it follows that $\lim _{\Lambda_{0} \rightarrow \infty} \partial_{y} V_{\Lambda, n}^{(l)}=0$; from the $y$-independence of the bound (1.25) one could easily repeat a proof analogous to (1.35) of the fact that the two limits commute; therefore the renormalized Green functions are independent of $S_{i r r}$. This independence is a manifestation of the large arbitrariness in the discretization of the field theory on the lattice.

Rotation-covariance of $\lim _{\Lambda_{0} \rightarrow \infty} V_{\Lambda, n}^{(l)}$ is proven inductively in the loop index, using the fact that in $(1.31-32)$ the coefficient functions $\tilde{D}_{z, \Lambda}$ are rotation-covariant.

We have shown therefore that $\lim _{\Lambda_{0} \rightarrow \infty} V_{\Lambda, n}^{(l)}\left(k_{1}, \ldots, k_{n-1}\right)$, with the renormalization conditions (1.21) are renormalized rotation-covariant Green functions, satisfying the bound (1.25), order by order in the loop expansion; furthermore they are independent of $S_{i r r}$. For $m \neq 0$ the Green functions $\lim _{\Lambda_{0} \rightarrow \infty} V_{0, n}^{(l)}\left(k_{1}, \ldots, k_{n-1}\right)$, with renormalization conditions (1.19), can be obtained from $\lim _{\Lambda_{0} \rightarrow \infty} V_{\Lambda, n}^{(l)}\left(k_{1}, \ldots, k_{n-1}\right)$ using (1.20) and therefore exist, are rotation-covariant and are independent of $S_{i r r}$. This completes the proof of renormalizability.

\subsection{Generalizations.}

Let us now relax the condition required previously on the tree-level two-point irrelevant terms. It is easy to take into account irrelevant terms with two legs at tree-level by including them in the kinetic term. Add for example the term

$$
\Delta S[\phi]=\frac{\xi}{2 \Lambda_{0}^{2}} \int_{k} \phi(-k)\left(\hat{k}^{2}\right)^{2} \phi(k)
$$

to the action $(1.4-5)$. The propagator replacing $(1.8)$ is then

$$
D_{\xi}(p)=\frac{1}{\hat{p}^{2}+m^{2}+\frac{\xi\left(\hat{p}^{2}\right)^{2}}{\Lambda_{0}^{2}}} .
$$

The parameter $\xi$ is chosen to satisfy $\xi>-\frac{\pi^{2}}{16}$, in such a way that

$$
\left|D_{\xi}(p)\right| \leq \frac{\beta}{\hat{p}^{2}+m^{2}}
$$

where $\beta$ is a positive constant.

In this case the propagator $D_{\xi}(p)$ has a single maximum in the Brillouin zone, and it coincides with $D(p)$ in the limit $\Lambda_{0} \rightarrow \infty$. 
The cut-off propagator is now defined as $D_{\Lambda, \xi}(p)=K_{\Lambda}(p) D_{\xi}(p)$, replacing (1.9). Then we can repeat the proof of renormalization along the lines of subsection 1.3, once we replace $D_{\Lambda}(p)$ with $D_{\Lambda, \xi}(p)$. It should be noticed that the bound (1.40) has been imposed in order to verify the induction hypothesis (1.25) at $s=0$, and that $\xi$ plays the role of $y$; for instance from

$$
\left\|\partial_{\xi} D_{\Lambda, \xi}\right\|_{\Lambda} \leq \frac{\alpha}{\Lambda_{0}^{2}}
$$

it follows that (1.25) holds for $s=0$, with $y$ replaced by $\xi$. Therefore in the continuum limit the renormalized Green functions do not depend on $\xi$.

A similar analysis holds for other two-point irrelevant terms. One can prove renormalizability provided (1.40) holds and the propagator has the usual continuum limit [3, 田

A last remark on the irrelevant terms is in order. In the formulation of subsection 1.3, $S_{i r r}$ is an assigned function satisfying (1.24). In some application the irrelevant terms are instead assigned as a function of the relevant terms, for instance one could be interested in an action containing

$$
c_{3} \phi^{4}+\frac{1}{\Lambda_{0}^{2}} c_{3} \phi^{6}+\ldots
$$

To prove renormalizability the procedure is the one used in point (iv. b) in subsection 1.3. One can easily show that the bound

$$
\left|c_{3}^{(l)}\right|<P\left(\ln \frac{\Lambda_{0}}{\Lambda_{1}}\right)
$$

holds. The rest of the induction proof then follows as in the previous subsection. 


\section{Renormalization of matter field theories on a lat- tice.}

\subsection{Renormalization of matter field theories on a lattice.}

The renormalization of a general class of matter field theories with a finite number of physical fields and of auxiliary fields on a space-time lattice (not necessarily hypercubic) can be done in a similar way to the one followed in the first section. We will not reproduce all the steps followed there; we will deal only with the points which are significantly different in the two cases; we will restrict our attention to rectangular lattices with lattice spacings $a_{1} \geq a_{2} \geq a_{3} \geq a_{4}$. We define $\hat{p}_{\mu}=\frac{2}{a_{\mu}} \sin \frac{p_{\mu} a_{\mu}}{2}$ and $\Lambda_{0}^{2}=\frac{\pi^{2}}{4} \sum_{\mu} a_{\mu}^{-2}$.

Consider a lattice theory in which there are fields $\Phi_{i}$ with canonical dimension $\Delta_{i}=$ $1, \frac{3}{2}$ or 2 , for scalars, fermions and auxiliary fields respectively. For semplicity all fields are chosen to be real. It is straightforward to generalize the analysis to the case of complex fields.

The action reads, in momentum space,

$$
S[\Phi]=\frac{1}{2} \int_{p} \sum_{i, j} \Phi_{i}(-p) D_{i j}^{-1}(p) \Phi_{j}(p)+S_{I}[\Phi]
$$

The matrix propagator is a periodic $C^{\infty}$ function on the Brillouin zone.

Introduce the propagator with infrared cut-off,

$$
D_{\Lambda, i j}(p)=D_{i j}(p) K_{\Lambda}(p)
$$

where $K_{\Lambda}$ is defined as after (1.9).

Assume that the propagator satisfies the following bounds:

$$
\left\|\partial_{\Lambda}^{w} \partial_{k}^{z} D_{\Lambda, i j}\right\|_{\Lambda} \leq \alpha \Lambda^{\Delta_{i}+\Delta_{j}-4-z-w}
$$

for $0<\Lambda \leq \Lambda_{0}$, and $\alpha$ a positive constant depending on $i, j, z$ and $w$.

$S_{I}$ contains the interaction terms, both relevant and irrelevant. The tree-level part of $S_{I}$ is at least cubic in the fields; each relevant tree-level term of $S_{I}$ has a parameter which is independent from $\Lambda_{0}$.

The irrelevant terms are chosen to be linear in the parameter $y$.

As in the first section, introduce the functional generator $V_{\Lambda}[\Phi]$ for the amputated connected Green functions (apart from the tree-level 2-point contribution); each graph contributing to these Green functions depends on $\Lambda$ only through its internal propagators $D_{\Lambda}$. Therefore $V_{\Lambda}$ satisfies a flow equation analogous to (1.16).

Indicate with $V_{\Lambda, I}$, where $I=i_{1}, \ldots, i_{n}$, the amputated Green function with external legs of fields $\Phi_{i_{1}}, \ldots, \Phi_{i_{n}}$. 
The amputated connected Green functions are defined as series in $\hbar, V_{\Lambda, I}=\sum_{l \geq 0} \hbar^{l} V_{\Lambda, I}^{(l)}$. The flux equation has the form

$$
\partial_{\Lambda} V_{\Lambda, I}^{(l)}=\frac{1}{2} \int_{p} \sum \partial_{\Lambda} D_{\Lambda, j k} V_{\Lambda, I, j k}^{(l-1)}+\sum \partial_{\Lambda} D_{\Lambda, j k} V_{\Lambda, I_{1}, j}^{\left(l_{1}\right)} V_{\Lambda, I_{2}, k}^{\left(l_{2}\right)}
$$

where $I_{1} \cup I_{2}=I$; for semplicity the momenta and the sums have not been written explicitly.

Differentiating the flow equation with respect to $y$ and $k$ we get the following inequalities, for $\Lambda_{1} \leq \Lambda \leq \Lambda_{0}$,

$$
\begin{aligned}
& \left\|\partial_{\Lambda} \partial_{y}^{v} \partial_{k}^{z} V_{\Lambda, I}^{(l)}\right\|_{\Lambda} \leq \sum d_{i j} \Lambda^{\Delta_{i}+\Delta_{j}-1}\left\|\partial_{y}^{v} \partial_{k}^{z} V_{\Lambda, I, i j}^{(l-1)}\right\|_{\Lambda} \\
& +\sum d_{z_{1}, z_{2}, z_{3}, I_{1}, I_{2}, i, j} \Lambda^{\Delta_{i}+\Delta_{j}-5-z_{3}}\left\|\partial_{y}^{v_{1}} \partial_{k}^{z_{1}} V_{\Lambda, I_{1}, i}^{\left(l_{1}\right)}\right\|_{\Lambda}\left\|\partial_{y}^{v_{2}} \partial_{k}^{z_{2}} V_{\Lambda, I_{2}, j}^{\left(l_{2}\right)}\right\|_{\Lambda}
\end{aligned}
$$

where $d_{i j}$ and $d_{z_{1}, z_{2}, z_{3}, I_{1}, I_{2}, i, j}$ are constants, independent of $\Lambda$ and $\Lambda_{0}$.

Impose the following renormalization conditions on the relevant terms:

$$
\partial_{k}^{z} V_{\Lambda_{1}, I}^{(l)}(0, \ldots, 0)=\alpha_{z, I}^{(l)}
$$

for $\sum_{s=1}^{n} \Delta_{i_{s}}+z \leq 4$.

These renormalization conditions generalize those in (1.21). If there are symmetries in the theory, which can be maintained at quantum level, one can impose relations among the renormalization constants present in (2.6); for instance in the case of the scalar theory discussed in the first section, the hypercubic symmetry and the discrete symmetry $\phi \rightarrow-\phi$ reduce the conditions (2.6) to the simpler form (1.21).

Assume that $S_{i r r}$ satisfies the following conditions:

$$
\begin{gathered}
\left\|\partial_{k}^{z} S_{\Lambda_{0}, n}^{(l)}\right\|_{\Lambda} \leq \frac{\Lambda^{5-z-\sum_{s=1}^{n} \Delta_{i_{s}}}}{\Lambda_{0}} P\left(\ln \frac{\Lambda_{0}}{\Lambda}\right), \quad l=0 \\
\left\|\partial_{k}^{z} S_{\Lambda_{0}, n}^{(l)}\right\|_{\Lambda} \leq \frac{\Lambda_{0}^{5-z-\sum_{s=1}^{n} \Delta_{i_{s}}}}{\Lambda_{0}} P\left(\ln \frac{\Lambda_{0}}{\Lambda_{1}}\right), \quad l>0 .
\end{gathered}
$$

These equations generalize (1.24).

Let us prove that for $\Lambda_{1} \leq \Lambda \leq \Lambda_{0}$ one has

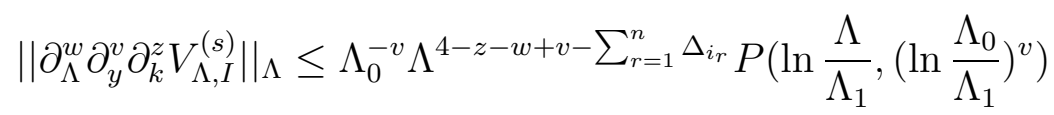

for any $s$, for $v, w=0,1, z \geq 0$ and $n \geq 1$.

We will prove (2.8) by induction in the loop index.

The assertion is true for $s=0$, as can be seen by the following power counting. 
Consider a tree level amputated connected graph contributing to $V_{\Lambda, I}^{(0)}$. Using the bounds (2.3) and observing that each factor $\Lambda^{\Delta_{i}}$ coming from an internal propagator is cancelled by a factor coming from a vertex, it follows that this graph is bound by const. $\times \Lambda^{b}$, with

$$
b=4(V-I)-\sum_{e x t} \Delta_{i}=4-\sum_{e x t} \Delta_{i}
$$

$\sum_{\text {ext }} \Delta_{i}$ is the sum of the canonical dimensions of the fields appearing in the external legs of the graph.

Differentiating $V_{\Lambda, I}^{(0)}$ with respect to $y$, the only non-vanishing contributions are those in which there is at least one irrelevant vertex, satisfying (2.7). Therefore $\left\|\partial_{y}^{v} V_{\Lambda, I}^{(0)}\right\|_{\Lambda} \leq$ $\Lambda^{b+v} \Lambda_{0}^{-v} P\left(\ln \frac{\Lambda_{0}}{\Lambda}\right)$.

It is easy to see that differentiating the tree level Green functions with respect to an external momentum, or with respect to $\Lambda$, lowers $b$ by one. Convergence of $\partial_{k}^{z} V_{\Lambda, I}^{(0)}$ for $\Lambda_{0} \rightarrow \infty$ follows trivially.

Therefore the induction hypothesis is true for $s=0$.

Assume that (2.8) is true for $s \leq l-1$; then we will show that (2.8) holds for $s=l$.

We can follow essentially the same steps as in the first section. Let us only point out a few differences.

The first step is the same, provided $n+z \geq 5$ is substituted by $\sum \Delta_{i}+z \geq 5$.

In the second step, in the inequality (2.5) the only terms in the RHS with $V_{\Lambda, I_{1}}^{(l)}$ are those with $n-1$ external legs. If (2.8) holds for $s=l$ up to $n-1$ external legs, then it holds for $s=l$, with $n$ external legs and $w=1$.

As in the first section, we prove (2.8) by induction in a sequence of points, each point being established by using the previous ones. This sequence consists in increasing the number of legs and going, for fixed number of legs, from irrelevant to relevant. It is easy to prove (2.8) for the tadpoles. The next point consists in proving (2.8) for $s=l, n=2$ and $\sum \Delta_{i}+z \geq 5$. The rest of the proof is quite similar to the one in the scalar case. Analogously one can prove convergence.

From (2.8) it follows that $\lim _{\Lambda_{0} \rightarrow \infty} \partial_{y} V_{\Lambda, I}^{(l)}=0$; therefore the renormalized Green functions are independent of $S_{i r r}$.

If the relevant part of $S_{I}$ is hypercubic-invariant and the pointwise limit of $D_{\Lambda i j}$ is $O(4)$-covariant in the limit $\Lambda_{0} \rightarrow \infty$, it is possible to choose the renormalization conditions in such a way that the renormalized Green functions are rotation covariant in the continuum limit. The proof of this fact is along the lines of a similar proof in Section 1. 


\section{Renormalization of fermionic models.}

In this section we will study the renormalizability of Yukawa theories. We will start by reviewing briefly the doubling problem and the Wilson fermions. Since the Wilson propagator satisfies the bound (2.3), it is straightforward to prove the renormalizability of Yukawa models with this kind of fermions, either using BPHZ [2, 3, 4] or the Polchinski approach here studied.

Staggered fermions [11] do not satisfy the bound (2.3), due to the presence of doublers at the border of the Brillouin zone. It has not yet been proven that interacting models with staggered fermions are renormalizable. We describe a simple kind of staggered fermions, which are staggered in one direction only, and have a single doubler [12, 13]. Although the fermionic propagator does not satisfy the bound (2.3), it satisfies a similar bound on a reduced Brillouin zone. Using this fact, we study a chiral sigma model on a hypercubic lattice, rewriting it equivalently on a reduced Brillouin zone and showing that it is renormalizable as a theory defined on a sublattice. Since renormalizability can be achieved maintaining the same translation symmetries as in the theory defined on the hypercubic lattice, renormalizability on the sublattice implies renormalizability of the chiral sigma model with this kind of staggered fermions on the hypercubic lattice.

The treatment of the flow equation with auxiliary fields, considered in the previous section, plays a crucial role in this proof of renormalizability.

We conjecture that the renormalizability of Yukawa models with Kogut-Susskind fermions can be proven in a similar way by studying them first on a hypercubic sublattice with lattice spacing $2 a$, on which the fermionic propagator satisfies the bound (2.3) 19.

\subsection{Naive fermions, the doubling problem and Wilson fermions.}

The naive fermionic action for massless fermions on a space-time hypercubic lattice is

$$
I_{0}=\frac{a^{3}}{2} \sum_{x} \sum_{\mu=1}^{4} \bar{\psi}_{x} \gamma_{\mu}\left(\psi_{x+\hat{\mu}}-\psi_{x-\hat{\mu}}\right) ;
$$

the gamma matrices are hermitian and satisfy $\left\{\gamma_{\mu}, \gamma_{\nu}\right\}=2 \delta_{\mu, \nu}$.

The inverse propagator for the naive fermionic action is

$$
S^{-1}(p)=i \sum_{\mu=1}^{4} \gamma_{\mu} \bar{p}_{\mu}
$$

where $\bar{p}_{\mu} \equiv \frac{1}{a} \sin \left(p_{\mu} a\right) ; S^{-1}(p)$ has zeroes for $\sin p_{\mu} a=0$, that is for $p_{\mu}=0, \frac{\pi}{a}$; it describes 16 Dirac fields in the continuum limit. $I_{0}$ shares this property with any bilinear 
and translationally invariant fermionic action, whose propagator satisfies the following properties [20]:

i) reflection $(\Theta)$ symmetry: $S^{-1}\left(p_{i}, p_{4}\right)=\gamma_{4} S^{-1 \dagger}\left(p_{i},-p_{4}\right) \gamma_{4}$;

ii) hypercubic space-time lattice symmetry, i.e. invariance under $\frac{\pi}{2}$ rotations of the coordinate axis;

iii) chiral symmetry: $S^{-1}(p)=-\gamma_{5} S^{-1}(p) \gamma_{5}$;

iv) locality, in the sense that $S^{-1}(p)$ is continuous with its first derivatives.

From (i) and (ii) it follows that $S^{-1}(p)=\gamma_{\mu} S^{-1 \dagger}\left(R_{\mu} p\right) \gamma_{\mu}$, where $R_{\mu}$ is the reflection operator on the $\mu$-th coordinate, $\left(R_{\mu} x\right)_{\nu}=\left(1-2 \delta_{\mu, \nu}\right) x_{\nu}$. From this fact and (iii) it follows that $S^{-1}(p)=-S^{-1}(-p)$, which together with periodicity $p_{\mu} \equiv p_{\mu}+\frac{2 \pi}{a}$ gives $S^{-1}(\bar{p})=0$ for $\bar{p}_{\mu}=0, \frac{\pi}{a}$; therefore a propagator satisfying these conditions propagates 16 modes.

This degeneracy is present also in the naive fermionic action for massive fermions.

Wilson [9] eliminated this degeneracy introducing a term which breaks the chiral symmetry in a hard way (since it has dimension 5),

$$
I_{W}=\frac{a^{3} r}{2} \sum_{x} \sum_{\mu=1}^{4} \bar{\psi}_{x}\left(2 \psi_{x}-\psi_{x+\hat{\mu}}-\psi_{x-\hat{\mu}}\right) .
$$

The Wilson action is site-reflection positive for $r=1$ [21] and it is link-reflection positive for $0<r \leq 1$ [22] ( for a review see 23] ). It describes one light mode and 15 massive modes, with masses of the order of $\frac{\pi}{a}$, which decouple in the continuum limit.

The propagator for the Wilson fermion is, in the massive case,

$$
S(p)=\frac{-i \sum_{\mu} \gamma_{\mu} \bar{p}_{\mu}+M(p)}{\bar{p}^{2}+M^{2}(p)}
$$

where

$$
M(p)=M+\frac{r a}{2} \hat{p}^{2}
$$

and $r$ is the Wilson parameter, satisfying $0<r \leq 1$. $M$ is the mass of the light fermionic mode. We recall that $\hat{p}_{\mu}=\frac{2}{a} \sin \frac{p_{\mu} a}{2}$.

The fermionic propagator becomes the Dirac propagator in the continuum limit $a \rightarrow 0$,

$$
\lim _{a \rightarrow 0} S(p)=\frac{1}{i \sum_{\mu} \gamma_{\mu} p_{\mu}+M}
$$

The denominator of $S(p)$ satisfies the bound

$$
\frac{1}{\bar{p}^{2}+M^{2}(p)} \leq \frac{r^{-2}}{\hat{p}^{2}+M^{2}}
$$


where $r$ is the Wilson parameter. To prove this inequality, observe that

$$
\begin{aligned}
\bar{p}^{2}+M^{2}(p) & =r^{2}\left(\hat{p}^{2}+M^{2}\right)+\left(1-r^{2}\right)\left[M^{2}+\bar{p}^{2}\right] \\
& +\frac{r^{2} a^{2}}{4}\left[\left(\hat{p}^{2}\right)^{2}-\sum_{\mu} \hat{p}_{\mu}^{4}\right]+r a M \hat{p}^{2} \geq r^{2}\left(\hat{p}^{2}+M^{2}\right)
\end{aligned}
$$

for $0<r \leq 1$ and $M \geq 0$.

It follows that the Wilson propagator satisfies the bound (2.3). Therefore, according to the previous section, one can construct renormalizable models using Wilson fermions.

\subsection{Fermions with minimal doubling.}

On a hypercubic space-time lattice, translation-invariance, locality, chiral symmetry and $\mathrm{CP} \Theta$ (charge-conjugation $\times$ parity $\times$ reflection) invariance of the action imply the existence of doubling of the Dirac modes on the lattice [8].

In order to have the minimal doubling allowed under these assumptions, either reflection symmetry or hypercubic invariance must be dropped.

In [12, 13] it has been given an example of lattice translation invariant and chirally symmetric fermionic action which breaks the hypercubic space-time symmetry to cubic symmetry and which has minimal doubling. The action is [13]

$$
I_{f}=I_{0}+\frac{i a^{3} \lambda}{2} \sum_{x} \sum_{\mu \neq 1} \bar{\psi}_{x} \gamma_{1}\left(2 \psi_{x}-\psi_{x+\hat{\mu}}-\psi_{x-\hat{\mu}}\right)+M a^{4} \sum_{x} \bar{\psi}_{x} \psi_{x}
$$

where for $M \neq 0$ the chiral symmetry

$$
\psi_{x} \rightarrow e^{i \beta \gamma_{5}} \psi_{x} \quad ; \quad \bar{\psi}_{x} \rightarrow \bar{\psi}_{x} e^{i \beta \gamma_{5}}
$$

is softly broken, since $\bar{\psi}_{x} \psi_{x}$ has dimension $3 ; I_{0}$ is the naive fermionic action (3.1).

The inverse propagator is

$$
S^{-1}(p)=i \sum_{\mu} \bar{p}_{\mu} \gamma_{\mu}+\frac{i a}{2} \lambda \hat{p}_{\perp}^{2} \gamma_{1}+M
$$

where $\hat{p}_{\perp}^{2} \equiv \sum_{\mu \neq 1} \hat{p}_{\mu}^{2}$. For $\lambda>1 / 2$ there are only two propagating modes, around $p=(0,0,0,0)$ and $p=\left(\frac{\pi}{a}, 0,0,0\right)$ [12]; in fact the inverse propagator is equal to $M$ provided $\sin p_{\mu} a=0$ for $\mu \neq 1$, which means $p_{\mu}=0, \frac{\pi}{a}$ for $\mu \neq 1$; and provided $\sin p_{1} a+$ $\lambda \sum_{\mu \neq 1}\left(1-\cos p_{\mu} a\right)=0$ which cannot be satisfied if $\lambda>1 / 2$ and $p_{\mu}=\frac{\pi}{a}$ for some $\mu \neq 1$. This is an example of fermions which are staggered in one direction only.

For $\frac{1}{2}<\lambda \leq 1$ the action (3.8) is also link-reflection positive, so that one can construct a transfer matrix over a Hilbert space of physical states 13 .

The hypercubic symmetry is broken to the cubic symmetry in the directions $x_{2}, x_{3}$ and $x_{4}$, which includes the axis-inversion symmetry $\psi_{x} \rightarrow i \gamma_{\mu} \gamma_{5} \psi_{R_{\mu} x}$, with $\mu \neq 1$. 
The action (3.8) has a discrete symmetry reflecting the fermion in its mirror fermion:

$$
\psi_{x} \rightarrow(-)^{x_{1} / a} \psi_{R_{1} x} \quad ; \quad \bar{\psi}_{x} \rightarrow(-)^{x_{1} / a} \bar{\psi}_{R_{1} x}
$$

The action is link-reflection invariant, that is, invariant under the antilinear mapping

$$
\Theta \psi_{\underline{x}, t}=\bar{\psi}_{\underline{x}, 1-t} \gamma_{4} \quad ; \quad \Theta \bar{\psi}_{\underline{x}, t}=\gamma_{4} \psi_{\underline{x}, 1-t}
$$

( it is also site-reflection invariant, but not site-reflection positive ). It is invariant under $C P$ transformations

$$
\psi_{\underline{x}, t} \rightarrow \gamma_{4} C \bar{\psi}_{\hat{1}-\underline{x}, t}^{T} ; \quad \bar{\psi}_{\underline{x}, t} \rightarrow-\psi_{\hat{1}-\underline{x}, t}^{T} C^{-1} \gamma_{4}
$$

where $C$ is the charge conjugation matrix. Therefore the action is $\mathrm{CP} \Theta$-invariant.

The propagator (3.10) does not satisfy the bound (2.3), since it is equal to $M^{-1}$ in the point $p=\left(\frac{\pi}{a}, 0,0,0\right)$. Similar problems arise with the Kogut-Susskind staggered fermions 19.

This difficulty can be overcome redefining the fermionic variables $\psi_{x}$ and $\bar{\psi}_{x}$, defined on the hypercubic lattice $\Xi$ in terms of new fermionic variables defined on the sublattice $\Xi^{\prime}$ characterized by $\frac{x_{1}}{a}$ even. Define, for $\frac{x_{1}}{a}$ even,

$$
\begin{aligned}
\Psi_{1, x}=\frac{1}{2}\left(\psi_{x}+\psi_{x+\hat{1}}\right) ; & \bar{\Psi}_{1, x}=\frac{1}{2}\left(\bar{\psi}_{x}+\bar{\psi}_{x+\hat{1}}\right) \\
\Psi_{2, x}=\frac{i}{2} \gamma_{1} \gamma_{5}\left(\psi_{x}-\psi_{x+\hat{1}}\right) ; \bar{\Psi}_{2, x} & =\frac{i}{2}\left(\bar{\psi}_{x}-\bar{\psi}_{x+\hat{1}}\right) \gamma_{1} \gamma_{5}
\end{aligned}
$$

This redefinition is similar to the one made in [19], where the Kogut-Susskind fermions are rewritten on a sublattice of lattice spacing $2 a$.

The fermionic action (3.8) becomes, in these variables,

$$
\begin{gathered}
I_{F}[\Psi, \bar{\Psi}]=2 a^{3} \sum_{x}^{\prime} \bar{\Psi}_{x}\left[\sum_{\mu \neq 1} \frac{1}{2} \gamma_{\mu} \otimes 1\left(\Psi_{x+\hat{\mu}}-\Psi_{x-\hat{\mu}}\right)+\frac{1}{4} \gamma_{1} \otimes 1\left(\Psi_{x+2 \hat{1}}-\Psi_{x-2 \hat{1}}\right)\right. \\
\left.-\frac{i}{4} \gamma_{5} \otimes \sigma_{1}\left(2 \Psi_{x}-\Psi_{x+2 \hat{1}}-\Psi_{x-2 \hat{1}}\right)+i \frac{\lambda}{2} \sum_{\mu \neq 1} \gamma_{1} \otimes \sigma_{3}\left(2 \Psi_{x}-\Psi_{x+\hat{\mu}}-\Psi_{x-\hat{\mu}}\right)+a M \Psi_{x}\right]
\end{gathered}
$$

where $\sum_{x}^{\prime}$ is the sum over $x \in \Xi^{\prime}$, and where $\Psi_{x}=\left(\Psi_{i, x}\right), i=1,2 . \sigma_{i}$ are the Pauli matrices.

The inverse propagator becomes

$$
\begin{gathered}
S^{-1}(p)=\Gamma+M= \\
i\left\{\sum_{\mu \neq 1} \gamma_{\mu} \otimes 1 \bar{p}_{\mu}+\frac{1}{2 a} \gamma_{1} \otimes 1 \sin \left(2 p_{1} a\right)-a \gamma_{5} \otimes \sigma_{1} \bar{p}_{1}^{2}+\frac{\lambda a}{2} \gamma_{1} \otimes \sigma_{3} \hat{p}_{\perp}^{2}\right\}+M
\end{gathered}
$$


which is periodic on the reduced Brillouin zone $\left|p_{1}\right| \leq \frac{\pi}{2 a},\left|p_{\mu}\right| \leq \frac{\pi}{a}, \mu \neq 1$. In the continuum limit this propagator becomes the standard Dirac propagator.

Let us show that this propagator satisfies the bound (2.3). Observe that

$$
\left\|K_{\Lambda} S\right\|_{\Lambda}=\left\|K_{\Lambda} \frac{\Gamma-M}{\Gamma^{2}-M^{2}}\right\|_{\Lambda} \leq c \Lambda\left\|K_{\Lambda} \frac{1}{\alpha 1+B}\right\|_{\Lambda}
$$

where

$$
\alpha=\bar{p}_{1}^{2}+\hat{p}_{\perp}^{2}+\frac{a^{2}}{4}\left[\left(\lambda \hat{p}_{\perp}^{2}\right)^{2}-\sum_{\mu \neq 1} \hat{p}_{\mu}^{4}\right]+M^{2}
$$

and

$$
B=a \lambda \hat{p}_{\perp}^{2}\left(1 \otimes \sigma_{3} \bar{p}_{1} \cos p_{1} a-a i \gamma_{1} \gamma_{5} \otimes \sigma_{2} \bar{p}_{1}^{2}\right)
$$

The smallest eigenvalue of $B$ is $\beta=-a \lambda \hat{p}_{\perp}^{2}\left|\bar{p}_{1}\right|$.

Therefore the minimum eigenvalue of $\alpha 1+B$ is

$$
\alpha+\beta \geq M^{2}+\bar{p}_{1}^{2}+\hat{p}_{\perp}^{2}\left(1-a \lambda\left|\bar{p}_{1}\right|\right)+\frac{a^{2}}{4}\left[\lambda^{2}\left(\hat{p}_{\perp}^{2}\right)^{2}-\sum_{\mu \neq 1} \hat{p}_{\mu}^{4}\right] .
$$

A detailed study of the RHS of (3.17) shows that in the range $\frac{1}{2}<\lambda \leq 1$ the following inequality holds:

$$
\alpha+\beta \geq M^{2}+\frac{1}{5}(2 \lambda-1)^{2}\left(\bar{p}_{1}^{2}+\hat{p}_{\perp}^{2}\right) .
$$

Using the norm (1.23) and the inequalities $\frac{4}{\pi^{2}} p^{2} \leq \bar{p}_{1}^{2}+\hat{p}_{\perp}^{2} \leq p^{2}$, for $p$ belonging to the reduced Brillouin zone, it follows that

$$
\left\|K_{\Lambda} \frac{1}{\alpha 1+B}\right\|_{\Lambda} \leq \text { const. } \times \Lambda^{-2}
$$

and therefore the propagator satisfies the bound (2.3) for $z=w=0$; it is easy to see that (2.3) is satisfied for any $z$ and $w$.

The action (3.15) has the link-reflection symmetry

$$
\Psi_{\underline{x}, t} \rightarrow \bar{\Psi}_{\underline{x}, 1-t} \gamma_{4} \otimes 1 ; \quad \bar{\Psi}_{\underline{x}, t} \rightarrow \gamma_{4} \otimes 1 \Psi_{\underline{x}, 1-t}
$$

It is invariant under the CP transformation

$$
\Psi_{\underline{x}, t} \rightarrow \gamma_{4} C \otimes \sigma_{3} \bar{\Psi}_{-\underline{x}, t} \quad ; \quad \bar{\Psi}_{\underline{x}, t} \rightarrow \Psi_{-\underline{x}, t}^{T} C^{-1} \gamma_{4} \otimes \sigma_{3}
$$

and it has the chiral symmetry (for $M=0$ )

$$
\Psi_{x} \rightarrow e^{i \beta \gamma_{5} \otimes \sigma_{3}} \Psi_{x} \quad ; \quad \bar{\Psi}_{x} \rightarrow \bar{\Psi}_{x} e^{i \beta \gamma_{5} \otimes \sigma_{3}}
$$

The discrete symmetry (3.11) takes the form

$$
\Psi_{x} \rightarrow \gamma_{1} \gamma_{5} \otimes \sigma_{2} \Psi_{R_{1} x} \quad ; \quad \bar{\Psi}_{x} \rightarrow-\bar{\Psi}_{R_{1} x} \gamma_{1} \gamma_{5} \otimes \sigma_{2}
$$

which mixes the $x_{1}$-inversion symmetry and the flavour symmetry. 


\subsection{Renormalizability of a chiral sigma model.}

Let us describe a sigma model on a hypercubic lattice, with two charged fermions and two neutral scalars, with a $U(1)_{V}$ vector symmetry and a $U(1)_{A}$ axial symmetry. The tree-level action is

$$
I^{(0)}=I_{f}[\psi, \bar{\psi}]+I_{B}[\sigma]+I_{B}[\pi]+a^{4} \sum_{x}\left[g_{1} \bar{\psi}_{x}\left(\sigma_{x}+i \gamma_{5} \pi_{x}\right) \psi_{x}+g_{2}\left(\sigma_{x}^{2}+\pi_{x}^{2}\right)^{2}\right]
$$

where $I_{f}[\psi, \bar{\psi}]$ is the free fermionic action (3.8) with $M=0$;

$$
I_{B}[\phi]=\frac{a^{2}}{2} \sum_{x, \mu} \phi_{x}\left(2 \phi_{x}-\phi_{x+\hat{\mu}}-\phi_{x-\hat{\mu}}\right)+a^{4} \sum_{x} \frac{m^{2}}{2} \phi_{x}^{2}
$$

is the free bosonic action for $\phi=\sigma, \pi . \sigma_{x}$ is a scalar, $\pi_{x}$ is a pseudoscalar.

The action (3.23) has the $U(1)_{V}$ phase symmetry $\psi_{x} \rightarrow e^{i \alpha} \psi_{x}, \quad \bar{\psi}_{x} \rightarrow e^{-i \alpha} \bar{\psi}_{x}$; the scalars are invariant under this symmetry. It has an axial symmetry $U(1)_{A}$; the chiral transformation on the fermions is given in (3.9); the scalars transform in the following way:

$$
\sigma_{x}+i \pi_{x} \rightarrow e^{-2 i \beta}\left(\sigma_{x}+i \pi_{x}\right)
$$

The action (3.23) has the discrete symmetry (3.11) on the fermions, and $\sigma_{x} \rightarrow$ $\sigma_{R_{1} x}, \quad \pi_{x} \rightarrow \pi_{R_{1} x}$ on the scalars; it is invariant under $\mathrm{CP}$ and under cubic space-time rotations and inversions in the $x_{2} x_{3} x_{4}$ directions. It has the link-reflection symmetry defined by (3.12) on the fermions, and by $\Theta \sigma_{\underline{x}, t} \rightarrow \sigma_{\underline{x}, 1-t}, \Theta \pi_{\underline{x}, t} \rightarrow \pi_{\underline{x}, 1-t}$ on the scalars.

The fermionic propagator does not satisfy the bound (2.3), so that it is not straighforward to prove the renormalizability of this model.

As in the previous subsection, we will rewrite this model on an anisotropic sublattice, in such a way that the fermion and its doubler become two fermions which are coupled by an irrelevant term, with propagator satisfying the bound (2.3). For consistency, it is also necessary to rewrite the scalars on this sublattice; a scalar splits in a propagating mode and in an auxiliary field. Define, for $x_{1}$ even,

$$
\begin{array}{r}
A_{x}=\frac{1}{2}\left(\sigma_{x}+\sigma_{x+\hat{1}}\right) \quad ; \quad B_{x}=\frac{1}{2}\left(\pi_{x}+\pi_{x+\hat{1}}\right) \\
F_{x}=\frac{1}{2} a^{-1}\left(\sigma_{x}-\sigma_{x+\hat{1}}\right) ; \quad G_{x}=\frac{1}{2} a^{-1}\left(\pi_{x}-\pi_{x+\hat{1}}\right)
\end{array}
$$

while the fermions are defined as in (3.14).

The free $A-F$ part of the bosonic action $I_{B}[\sigma]$ becomes, on the sublattice $\Xi^{\prime}$,

$$
I_{q u a d r}[A, F]=2 a^{4} \sum_{x}^{\prime}\left\{\frac{1}{4 a^{2}} A_{x}\left(2 A_{x}-A_{x+2 \hat{1}}-A_{x-2 \hat{1}}\right)+\frac{1}{4} F_{x}\left(6 F_{x}+F_{x+2 \hat{1}}+F_{x-2 \hat{1}}\right)\right.
$$




$$
\begin{gathered}
+\frac{1}{2 a} F_{x}\left(A_{x+2 \hat{1}}-A_{x-2 \hat{1}}\right)+\frac{1}{2 a^{2}} \sum_{\mu \neq 1}\left(A_{x}\left(2 A_{x}-A_{x+\hat{\mu}}-A_{x-\hat{\mu}}\right)\right. \\
\left.\left.+a^{2} F_{x}\left(2 F_{x}-F_{x+\hat{\mu}}-F_{x-\hat{\mu}}\right)\right)+\frac{m^{2}}{2}\left(A_{x}^{2}+a^{2} F_{x}^{2}\right)\right\}
\end{gathered}
$$

The interaction term is, at tree level,

$$
\begin{aligned}
I_{i n t}^{(0)}= & 2 a^{4} \sum_{x}^{\prime}\left\{g_{1} \bar{\Psi}_{x}\left[\left(A_{x}+i \gamma_{1} \gamma_{5} \otimes \sigma_{1} a F_{x}\right)+i \gamma_{5} \otimes \sigma_{3}\left(B_{x}+i \gamma_{1} \gamma_{5} \otimes \sigma_{1} a G_{x}\right)\right] \Psi_{x}\right. \\
& \left.+g_{2}\left[\left(A_{x}^{2}+B_{x}^{2}+a^{2} F_{x}^{2}+a^{2} G_{x}^{2}\right)^{2}+4 a^{2}\left(A_{x} F_{x}+B_{x} G_{x}\right)^{2}\right]\right\}
\end{aligned}
$$

The tree-level action (3.23) is equivalent to

$$
I^{(0)}=I[A, F]+I[B, G]+I_{F}[\Psi, \bar{\Psi}]+I_{i n t}^{(0)}
$$

The translation transformation by one site in the direction $x_{1}$ on $\Xi$ become, on $\Xi^{\prime}$,

$$
\begin{aligned}
A_{x} & \rightarrow \frac{1}{2}\left(A_{x+2 \hat{1}}+A_{x}\right)+\frac{a}{2}\left(F_{x+2 \hat{\imath}}-F_{x}\right) \\
F_{x} & \rightarrow-\frac{1}{2}\left(F_{x+2 \hat{1}}+F_{x}\right)-\frac{1}{2 a}\left(A_{x+2 \hat{1}}-A_{x}\right) \\
\Psi_{x} & \rightarrow 1 \otimes \sigma_{3}\left[\frac{1-i \gamma_{1} \gamma_{5} \otimes \sigma_{1}}{2} \Psi_{x}+\frac{1+i \gamma_{1} \gamma_{5} \otimes \sigma_{1}}{2} \Psi_{x+2 \hat{1}}\right] \\
\bar{\Psi}_{x} & \rightarrow\left[\bar{\Psi}_{x} \frac{1-i \gamma_{1} \gamma_{5} \otimes \sigma_{1}}{2}+\bar{\Psi}_{x+2 \hat{1}} \frac{1+i \gamma_{1} \gamma_{5} \otimes \sigma_{1}}{2}\right] 1 \otimes \sigma_{3}
\end{aligned}
$$

and similarly for $B_{x}$ and $G_{x}$. All these symmetries are preserved in presence of the cut-off $K_{\Lambda}(p)=K\left(\frac{\hat{p}_{\perp}^{2}+\bar{p}_{1}^{2}}{\Lambda^{2}}\right)$, introduced for all the propagators. Therefore they are compatible with the flow equation. Choosing appropriately the renormalization conditions on the relevant terms and the boundary conditions on the irrelevant terms, it follows that the connected Green functions $V_{\Lambda}^{(l)}$ have the translation symmetry (3.28) for any $\Lambda$.

Modulo irrelevant terms, the most general quadratic scalar term in the sector $A-F$ of the bare action, which is invariant under the transformation (3.28), has the form

$$
\begin{aligned}
I_{A, F}^{q u a d r} & =2 a^{4} \sum_{x}^{\prime}\left\{\frac{\alpha_{1}}{2}\left(A_{x}^{2}+a^{2} F_{x}^{2}\right)+\frac{\alpha_{2}+\alpha_{3}}{4 a^{2}} A_{x}\left(2 A_{x}-A_{x+2 \hat{1}}-A_{x-2 \hat{1}}\right)+2 \alpha_{2} F_{x}^{2}\right. \\
& +\frac{\alpha_{3}-\alpha_{2}}{4} F_{x}\left(2 F_{x}-F_{x+2 \hat{1}}-F_{x-2 \hat{1}}\right)+\frac{\alpha_{2}}{2 a} F_{x}\left(A_{x+2 \hat{1}}-A_{x-2 \hat{1}}\right) \\
+ & \left.\frac{\alpha_{4}}{2 a^{2}} \sum_{\mu \neq 1}\left(A_{x}\left(2 A_{x}-A_{x+\hat{\mu}}-A_{x-\hat{\mu}}\right)+a^{2} F_{x}\left(2 F_{x}-F_{x+\hat{\mu}}-F_{x-\hat{\mu}}\right)\right)\right\}
\end{aligned}
$$

Due to the axial symmetry the corresponding term in the $B-G$ sector has the same coefficients $\alpha_{1}, \alpha_{2}, \alpha_{3}, \alpha_{4}$.

The same arguments lead to the quadratic fermionic term of the bare action of the form

$$
\begin{aligned}
I_{\Psi \Psi}^{\text {quadr }}= & 2 a^{4} \sum_{x}^{\prime} \bar{\Psi}_{x}\left[\beta_{1} \sum_{\mu \neq 1} \frac{1}{2 a} \gamma_{\mu} \otimes 1\left(\Psi_{x+\hat{\mu}}-\Psi_{x-\hat{\mu}}\right)+\beta_{2} \frac{1}{4 a} \gamma_{1} \otimes 1\left(\Psi_{x+2 \hat{1}}-\Psi_{x-2 \hat{1}}\right)\right. \\
& \left.-\frac{i}{4 a} \beta_{2} \gamma_{5} \otimes \sigma_{1}\left(2 \Psi_{x}-\Psi_{x+2 \hat{1}}-\Psi_{x-2 \hat{1}}\right)+i \beta_{3} \gamma_{1} \otimes \sigma_{3} \Psi_{x}+\beta_{4} \Psi_{x}\right]
\end{aligned}
$$


The bare interaction term has the same form as in (3.27), with the coupling constants $g_{1}$ and $g_{2}$ substituted by two bare parameters $\chi_{1}$ and $\chi_{2}$. Therefore starting with the bare lagrangian the renormalization procedure will require 10 independent conditions, at any loop order.

A parametrization of the two-point solution of the flux equation in the $A-F$ sector having the bare action just discussed as boundary condition is

$$
\begin{array}{r}
V_{A A}^{\Lambda}=\Delta_{+}^{\Lambda}(p)+\Delta_{-}^{\Lambda}(p) \cos p_{1} a ; \quad V_{F F}^{\Lambda}=a^{2}\left(\Delta_{+}^{\Lambda}(p)-\Delta_{-}^{\Lambda}(p) \cos p_{1} a\right) \\
V_{F A}^{\Lambda}=-i a \Delta_{-}^{\Lambda}(p) \sin p_{1} a
\end{array}
$$

where $\Delta_{+}^{\Lambda}(p)$ and $\Delta_{-}^{\Lambda}(p)$ have period $\frac{2 \pi}{a}$ in the directions $p_{2}, p_{3}, p_{4}$ and are, respectively, periodic and antiperiodic in the direction $p_{1}$, with period $\frac{\pi}{a}$. They correspond to $\frac{1}{4}\left[V_{\sigma \sigma}^{\Lambda}\left(p_{1}, ..\right)+V_{\sigma \sigma}^{\Lambda}\left(p_{1}+\frac{\pi}{a}, ..\right)\right]$ and $\frac{1}{4}\left[V_{\sigma \sigma}^{\Lambda}\left(p_{1}, ..\right)-V_{\sigma \sigma}^{\Lambda}\left(p_{1}+\frac{\pi}{a}, ..\right)\right]$, where $V_{\sigma \sigma}^{\Lambda}$ is the twopoint amputated connected Green function on the lattice $\Xi$.

Equation (3.29) can be obtained from (3.31) with a suitable choice of $\Delta_{+}$and $\Delta_{-}$at $\Lambda=\Lambda_{0}$.

Equation (3.31) leads to the following renormalization conditions

$$
\begin{aligned}
& V_{A A}^{\Lambda_{1}(l)}(0)=\alpha_{1}^{\prime(l)} ; \quad \frac{d^{2}}{d p_{1}^{2}} V_{A A}^{\Lambda_{1}(l)}(0)=4\left(\alpha_{2}^{\prime(l)}+\alpha_{3}^{\prime(l)}\right) ; \quad \frac{d^{2}}{d p_{k}^{2}} V_{A A}^{\Lambda_{1}(l)}(0)=2 \alpha_{4}^{\prime(l)} \\
& V_{F A}^{\Lambda_{1}(l)}(0)=0 ; \quad V_{F F}^{\Lambda_{1}(l)}(0)=4 \alpha_{2}^{\prime(l)}+a^{2} \alpha_{1}^{\prime(l)} ; \frac{d}{d p_{k}} V_{F A}^{\Lambda_{1}(l)}(0)=0 \quad, k \neq 1 \\
& \frac{d}{d p_{1}} V_{F A}^{\Lambda_{1}(l)}(0)=2 i \alpha_{2}^{\prime(l)}
\end{aligned}
$$

Analogous renormalization conditions are imposed in the $B-G$ sector with the same coefficients $\alpha_{1}^{\prime(l)}, \ldots, \alpha_{4}^{\prime(l)}$. The irrelevant terms in (3.32) are kept to preserve the symmetry (3.28).

The renormalization conditions consistent with (3.9), (3.11-13), (3.28) and with the axial reflection symmetry assign the following expansion of $V_{\bar{\Psi} \Psi}$ in the neighborhood of $p=0$ :

$$
V_{\bar{\Psi} \Psi}^{\Lambda_{1}(l)}(p)=2 \beta_{1}^{\prime(l)} \sum_{k} p_{k} \gamma_{k}+2\left(\beta_{2}^{\prime(l)} p_{1}+i \beta_{3}^{\prime(l)}\right) \gamma_{1} \otimes \sigma_{3}+O\left(p^{2}\right)
$$

For the three-point functions the renormalization conditions are

$$
\begin{aligned}
& V_{\bar{\Psi} \Psi A}^{\Lambda_{1}(l)}(0)=\chi_{1}^{\prime(l)} ; V_{\bar{\Psi} \Psi B}^{\Lambda_{1}(l)}(0)=i \gamma_{5} \otimes \sigma_{3} \chi_{1}^{\prime(l)} \\
& V_{A^{4}}^{\Lambda_{1}(l)}(0)=\chi_{2}^{\prime(l)} ; \quad V_{B^{4}}^{\Lambda_{1}(l)}(0)=\chi_{2}^{\prime(l)} ; \quad V_{A^{2} B^{2}}^{\Lambda_{1}(l)}(0)=\frac{\chi_{2}^{\prime(l)}}{3}
\end{aligned}
$$

As the fermionic propagators, also the bosonic propagators associated to (3.26) satisfy equation (2.3), with $\Delta_{A}=\Delta_{B}=1$ and $\Delta_{F}=\Delta_{G}=2$; therefore the model is renormalizable on the lattice $\Xi^{\prime}$. 


\subsection{Back to the hypercubic lattice.}

We consider now the relation between the renormalization on the cubic lattice $\Xi^{\prime}$ and the hypercubic lattice $\Xi$.

This relation has some non-trivial feature already in the case of a pure scalar theory. Let us consider a scalar theory on $\Xi$, which is anisotropic in the $x_{1}$ direction. Then one must impose three independent renormalization conditions on the two-point function at every loop order. Considering instead the corresponding theory on the lattice $\Xi^{\prime}$, the scalar field is replaced by a scalar field and an auxiliary field, as in subsection 3.3; one must impose four independent renormalization conditions, as in (3.32). On $\Xi^{\prime}$ there is one renormalization condition which is not necessary on $\Xi$; in fact $V_{F F}^{\Lambda_{1}(l)}(0)=4 \alpha_{2}^{\prime(l)}+a^{2} \alpha_{1}^{\prime(l)}$ corresponds to

$$
V_{\Lambda_{1}, \phi \phi}^{(l)}\left(\frac{\pi}{a}, 0,0,0\right)=\frac{8}{a^{2}} \alpha_{2}^{\prime(l)}+2 \alpha_{1}^{\prime(l)}
$$

This extra renormalization condition, which is a requirement on the behavior of the twopoint function at the border of the Brillouin zone, is allowed by the fact that the $\alpha_{2}$-term and the $\alpha_{3}$-term in the action (3.29) are proportional, modulo an irrelevant term which on $\Xi$ has the form

$$
\int_{p} \phi(-p) \phi(p)\left(\hat{p}_{1}^{2}-\bar{p}_{1}^{2}\right) .
$$

Observe that this functional, which is irrelevant on $\Xi$, is transformed into a relevant operator on $\Xi^{\prime}$; in fact the limit $p_{1} \rightarrow \frac{\pi}{a}$ on $\Xi$ corresponds to the limit $p_{1} \rightarrow 0$ on $\Xi^{\prime}$ and in this limit this operator does not vanish. Therefore renormalizability of the scalar theory on $\Xi^{\prime}$ is a stronger requirement than renormalizability on $\Xi$.

Let us show that for the chiral sigma model the renormalizability on $\Xi^{\prime}$ implies the renormalizability on $\Xi$. Consider on $\Xi^{\prime}$ a component of the Volterra expansion of $V$,

$$
\int_{B / 2} \ldots \int_{B / 2} \delta_{B / 2}\left(\sum p\right) \Phi_{I_{1} \ldots} \Phi_{I_{n}} V_{I_{1}, \ldots, I_{n}}
$$

where $\Phi_{I}$ stands for the fields $A, B, F, G, \Psi, \bar{\Psi} . \int_{B / 2}$ stands for the integral on Brillouin zone corresponding to $\Xi^{\prime} ; \delta_{B / 2}$ is the corresponding Dirac delta-function of momentum conservation. Using equations (3.14) and (3.25) in momentum space, the amputated Green functions on $\Xi$ will be suitable linear combinations of the amputated Green functions on $\Xi^{\prime}$. To show this, consider for example the following manipulation in (3.36),

$$
\begin{aligned}
\int_{0}^{\frac{\pi}{a}} d p_{1} \ldots \Psi_{1}\left(p_{1}, \ldots\right)= & 2^{-\frac{1}{2}} \int_{0}^{\frac{\pi}{a}} . .\left[\psi\left(p_{1}, \ldots\right)\left(1+e^{-i p_{1} a}\right)+\psi\left(p_{1}-\frac{\pi}{a}, \ldots\right)\left(1-e^{-i p_{1} a}\right)\right] \\
& =\int_{\frac{-\pi}{a}}^{\frac{\pi}{a}} d p_{1} \ldots \psi\left(p_{1}, \ldots\right)\left(1+e^{-i p_{1} a}\right)
\end{aligned}
$$


where we have used the periodicity of $\frac{\pi}{a}$ for the terms not explicitly written. The integrals over the momenta $p_{\perp}$ is understood. Analogously

$$
\begin{gathered}
\int_{0}^{\frac{\pi}{a}} d p_{1} \ldots \Psi_{2}\left(p_{1}, \ldots\right)=2^{-\frac{1}{2}} \int_{-\frac{\pi}{a}}^{\frac{\pi}{a}} d p_{1} \ldots i \gamma_{1} \gamma_{5} \psi\left(p_{1}, \ldots\right)\left(1-e^{-i p_{1} a}\right) \\
\int_{0}^{\frac{\pi}{a}} d p_{1} \ldots A\left(p_{1}, \ldots\right)=2^{-\frac{1}{2}} \int_{-\frac{\pi}{a}}^{\frac{\pi}{a}} d p_{1} \ldots \sigma\left(p_{1}, \ldots\right)\left(1+e^{-i p_{1} a}\right) \\
\int_{0}^{\frac{\pi}{a}} d p_{1} \ldots F\left(p_{1}, \ldots\right)=2^{-\frac{1}{2}} \int_{-\frac{\pi}{a}}^{\frac{\pi}{a}} d p_{1} \ldots a^{-1} \sigma\left(p_{1}, \ldots\right)\left(1-e^{-i p_{1} a}\right)
\end{gathered}
$$

and similarly for $B, G, \pi$. Using $(3.37-40)$ for any $p_{1}$-integration variable, (3.36) can be transformed in a multiple integral over the full Brillouin zone.

The delta-function of momentum conservation on the reduced Brillouin zone can be expressed in terms of the delta-function on the full Brillouin zone,

$$
\delta_{B / 2}\left(p_{1}, p_{2}, p_{3}, p_{4}\right)=\delta_{B}\left(p_{1}, p_{2}, p_{3}, p_{4}\right)+\delta_{B}\left(p_{1}+\frac{\pi}{a}, p_{2}, p_{3}, p_{4}\right)
$$

This last formula shows that in general, due to the presence of the translated deltafunction, equations $(3.36-40)$ do not lead to the Green functions of a theory on $\Xi$. But in our case, due to the imposition of the symmetry (3.28), this actually happens, indeed in the sum all the terms containing the translated delta-function must add to zero. Therefore we can conclude that every term (3.36) leads to a contribution to the Green functions of the hypercubic lattice $\Xi$ convergent in the continuum limit.

Moreover, (3.38) shows that, concerning the fermionic lines, not only the "naive limit' $a \rightarrow 0$ of the Green functions on $\Xi$ at fixed momenta exists, but also the limit

$\lim _{a \rightarrow 0} V_{\psi . .}\left(p_{1}+\frac{\pi}{a}, ..\right)$ exists and it is not vanishing; its physical interpretation is of course the existence of a second fermionic particle.

\section{$3.5 O(4)$ invariance.}

In order to get an easy proof of the $O(4)$ invariance of the theory in the continuum limit, a further change of the field variables is suitable. Indeed on $\Xi^{\prime}$ the pointwise limit for $a \rightarrow 0$ of the propagator in the scalar sector is not manifestly $O(4)$-invariant, as can be seen by Fourier-transforming equation (3.26).

The amputated Green functions of the field $\sigma$ on $\Xi$ have contributions from both the Green functions with $A$ and $F$ external legs; thus only in the sum, in the continuum limit, the symmetry will be restored. 
Let us make the redefinitions

$$
A(x)=A^{\prime}(x) \quad ; \quad F(x)=F^{\prime}(x)-\frac{1}{8 a}\left(A_{x+2 \hat{1}}^{\prime}-A_{x-2 \hat{1}}^{\prime}\right)
$$

Analogously for the fields $B$ and $G$. In terms of the new fields equation (3.26) in momentum space becomes, up to irrelevant terms,

$$
I\left[A^{\prime}, F^{\prime}\right]=\frac{1}{2} \int_{B / 2} \frac{d^{4} p}{(2 \pi)^{4}}\left[A^{\prime}(p) A^{\prime}(-p)\left(\hat{p}_{\perp}^{2}+\bar{p}_{1}^{2}+m^{2}\right)+4 F^{\prime}(p) F^{\prime}(-p)\right]
$$

Moreover $F^{\prime}$ and $G^{\prime}$ (as $F$ and $G$ before) appear in the interaction part of the action only in the irrelevant terms. From this fact one can easily show that in the continuum limit the fields $F^{\prime}$ and $G^{\prime}$ decouple, in the sense that, up to the $F^{\prime}-F^{\prime}$ and the $G^{\prime}-G^{\prime}$ twopoint terms, all the interaction amputated Green functions with some $F^{\prime}$ and $G^{\prime}$ external legs vanish. To show this, let us multiply by a parameter $y$ all the interaction terms in the bare lagrangian which depend on $F^{\prime}$ and $G^{\prime}$. For $y=1$ the action is equivalent to the one on the hypercubic lattice $\Xi$, while for $y=0$ this is not true but the fields $F^{\prime}$ and $G^{\prime}$ trivially decouple. In general, for $y \neq 1$, renormalization requires the imposition of the renormalization conditions on all the terms with dimension lower or equal to 4 . However we choose to impose again the conditions corresponding to (3.32), (3.33) and (3.34). The theory defined in this way is the original one for $y=1$; after taking the limit $a \rightarrow 0$ the solution of the flow equation does not depend on $y$, as it was shown in subsection 1.3, and in the remark in subsection 1.4; they are in particular equal to those of the $y=0$ case.

We showed in subsection 1.3 that in the limit $a \rightarrow 0$ the solutions of the flux equation satisfy the system of integral equations $(1.31-32)$. Now we have a system of equations involving functions with only $A^{\prime}, B^{\prime}, \Psi, \bar{\Psi}$ external legs, while the two-point functions of the auxiliary fields are momentum-independent.

Only $O(4)$-invariant propagators appear and then, as in subsection 1.3, we conclude that with a suitable choice of the constants of the renormalization conditions (for instance all vanishing for $l \neq 0$ ) the continuum limit of the solutions of the flux equations on $\Xi^{\prime}$ are $O(4)$-covariant. Thus from equations $(3.37-39)$, the terms on $\Xi$ coming from the functions on $\Xi^{\prime}$ with $A^{\prime}, B^{\prime}, \Psi, \bar{\Psi}$ legs will be $O(4)$-covariant; from $(3.40) V_{F^{\prime} F^{\prime}}$ could contribute only to $V_{\sigma \sigma}$, but an explicit computation shows that in the continuum limit this contribution vanishes. Therefore $O(4)$-invariance on $\Xi$ is proven for an arbitrary choice of the extra renormalization parameter discussed in the previous subsection. The renormalized Green functions do not depend on this parameter.

In this section we have studied a chiral sigma model, which has massless particles; due to the infrared divergences it is by no means trivial to show that the existence of $V_{\Lambda_{1}}$ implies that of the functional $V$ at $\Lambda=0$. In the formalism of the flux equation (on the continuum) this problem has been studied in 18, 24]. 


\section{Conclusions.}

In this paper we have shown that the Polchinski approach to renormalization can be applied on the lattice. Using this method we have proved the renormalizability of the scalar and Yukawa models on the lattice with Wilson fermions. A proof of these facts has been obtained previously by Reisz [2, 3, 4] using BPHZ. We have also treated a Yukawa model with a simple kind of staggered fermions. We proved that it is renormalizable provided a renormalization condition on an irrelevant term is added to the usual renormalization conditions; this extra condition is related to the two-point scalar Green function at the point on the border of the Brillouin zone in which there is the doubler pole of the fermionic propagator. While we have shown that such a renormalization procedure is sufficient to prove the renormalizability, we have not shown that it is necessary. Since the corresponding counterterm (3.35) does not appear in the one-loop bare action, only from a three-loop computation on the hypercubic lattice one might see if this extra renormalization condition is necessary.

It would be interesting to investigate the renormalizability of models with KogutSusskind staggered fermions along the same lines on this paper. For instance in the case of four flavours (that is in the case of one naive fermion) we expect that a proof of renormalizability can be given, involving fifteen extra renormalization conditions, related to the two-point scalar Green function at the points on the border of the Brillouin zone in which there are the fermionic doublers. 


\section{References}

[1] K.G. Wilson, Phys. Rev. D10 (1974) 2445.

[2] T. Reisz, Comm. Math. Phys. 116 (1988) 81; 116 (1988) 573.

[3] T. Reisz, Comm. Math. Phys. 117 (1988) 79; 117 (1988) 639.

[4] T. Reisz, Nucl. Phys. B318 (1989) 417.

[5] N.N. Bogoliubov and D. Shirkov, Introduction to the theory of quantized fields (Wiley/Interscience, New York).

[6] For a recent review see V. Rivasseau, From perturbative to constructive renormalization (Princeton University Press, Princeton, 1991).

[7] H.B. Nielsen and M. Ninomiya, Nucl. Phys. B185 (1981) 20; Erratum B195 (1982) 541.

[8] L. H. Karsten, Phys. Lett. 104B (1981) 315.

[9] K.G. Wilson, 'Quarks and strings on a lattice', in New Phenomena in Subnuclear Physics, ed. A. Zichichi, Part A (1975) 69.

[10] M. Lüscher, 'Selected topics in lattice field theory', in Fields, Strings and Critical Phenomena, Les Houches (1988).

[11] J. Kogut and L. Susskind, Phys. Rev. D11 (1975) 395;

L.Susskind, Phys. Rev. D16 (1977) 3031.

[12] F. Wilczek, Phys. Rev. Lett. 59 (1987) 2397.

[13] M. Pernici, Phys. Lett. B346 (1995) 99.

[14] J. Polchinski, Nucl. Phys. B231 (1984) 269.

[15] K. G. Wilson, Phys. Rev. B4 (1971) 3174, 3184;

K. G. Wilson and J.G. Kogut, Phys. Rep. 12 (1974) 75.

[16] G. Keller, C. Kopper and M. Salmhofer, Helv. Phys. Acta 65 (1992) 32;

R.D. Ball and R.S. Thorne, Ann. Phys. 236 (1994) 117.

[17] C. Becchi, 'On the construction of renormalized quantum field theory using renormalization group techniques', in Elementary particles, Quantum Fields and Statistical Mechanics, eds. M. Bonini, G. Marchesini, E. Onofri, Parma University 1993. 
[18] M. Bonini, M. D’Attanasio and G. Marchesini, Nucl. Phys. B409 (1993) 441.

[19] H. Kluberg-Stern, A. Morel, O. Napoly and B. Petersson, Nucl. Phys. B220 [FS8] (1983) 447.

[20] A. Pelissetto, Ann. Phys. (N.Y) 182 (1988) 177.

[21] M. Lüscher, Comm. Math. Phys. 54 (1977) 283.

[22] K. Osterwalder and E. Seiler, Ann. Phys. (N.Y.) 110 (1978) 440.

[23] I. Montvay and G. Münster, Quantum fields on a lattice (Cambridge University Press, 1994).

[24] G. Keller and C. Kopper, Comm. Math. Phys. 161 (1994) 515;

R.D. Ball and R.S. Thorne, 'Infrared and ultraviolet behaviour of effective scalar field theory' CERN-TH-7233-94. 